\title{
THE NEWTON POLYGON OF A RATIONAL PLANE CURVE
}

\author{
CARLOS D'ANDREA AND MARTÍN SOMBRA
}

\begin{abstract}
We show that the Newton polygon of the implicit equation of a rational plane curve is explicitly determined by the multiplicities of any of its parametrizations. As an application, we determine the Newton polygon of a curve parameterized by generic Laurent polynomials or by generic rational functions, with explicit genericity conditions. We also study the variety of rational curves with given Newton polygon, and show in particular that any convex lattice polygon with positive area is the Newton polygon of a rational plane curve.
\end{abstract}

\section{INTRODUCTION}

Rational plane curves can be represented either by a parametrization or by their implicit equation. In the present text we focus on the interplay between both representations. Our aim is to show that the Newton polygon of the implicit equation of a rational plane curve is determined by the multiplicities of any given parametrization and viceversa, that these multiplicities are determined up to a certain extent by the Newton polygon of the curve. This correspondence is explicit and we show that the polygon can be computed algorithmically.

Let $\mathbb{K}$ be an algebraically closed field and set $\mathbb{T}^{n}:=\left(\mathbb{K}^{\times}\right)^{n}$ for the $n$-dimensional algebraic torus. For a plane curve $C \subset \mathbb{T}^{2}$ we consider its defining equation $E_{C} \in$ $\mathbb{K}\left[x^{ \pm 1}, y^{ \pm 1}\right]$, which is an irreducible Laurent polynomial well-defined up to a monomial factor. We will be mostly interested in the Newton polygon of $C$

$$
\mathrm{N}(C) \subset \mathbb{R}^{2},
$$

defined as the convex hull of the exponents in the monomial expansion of $E_{C}$. This is a lattice convex polygon in the sense that its vertexes lie in the lattice $\mathbb{Z}^{2}$; it is well-defined up to a translation. Note that $\mathrm{N}(C)$ might reduce to a segment.

On the other hand, let $f, g \in \mathbb{K}(t)^{\times}$be rational functions which are not simultaneously constant and consider the map

$$
\rho: \mathbb{T}^{1} \rightarrow \mathbb{T}^{2} \quad, \quad t \mapsto(f(t), g(t)) .
$$

The Zariski closure $\overline{\rho\left(\mathbb{T}^{1}\right)}$ of its image is a rational curve in $\mathbb{T}^{2}$. We denote by $\operatorname{deg}(\rho) \in$ $\mathbb{N} \backslash\{0\}$ the degree of $\rho$, that is the cardinality of the fiber of $\rho$ above a generic point in its image.

Date: March 3, 2019.

2000 Mathematics Subject Classification. Primary 14H50; Secondary 14Q05, 14C17, 52B20.

Key words and phrases. Rational plane curve, parametrization, implicit equation, Newton polygon, mixed integral.

Both authors were supported by the Programa Ramón y Cajal of the Ministerio de Educación y Ciencia, Spain, and by the research projects MTM2007-67493 (D'Andrea) and MTM200614234 (Sombra). 
Let $\mathbb{P}^{1}$ denote the projective line over $\mathbb{K}$, then for each $v \in \mathbb{P}^{1}$ we consider the multiplicity of $\rho$ at $v$ defined as

$$
\left.\operatorname{ord}_{v}(\rho):=\operatorname{ord}_{v}(f), \operatorname{ord}_{v}(g)\right) \in \mathbb{Z}^{2}
$$

where $\operatorname{ord}_{v}(h)$ denotes the order of vanishing of $h$ at the point $v$. Note that $\operatorname{ord}_{v}(\rho)=$ $(0,0)$ for all but a finite number of $v$ 's and that the introduced family of vectors satisfies the balancing condition

$$
\sum_{v \in \mathbb{P}^{1}} \operatorname{ord}_{v}(\rho)=(0,0)
$$

because the sum of the order of the zeros and poles of a rational function is zero.

Given a family $B$ of vectors of $\mathbb{Z}^{2}$ which are zero except for a finite number of them and satisfy the balancing condition, we denote by $P(B)$ the lattice convex polygon constructed by rotating -90 degrees the non-zero vectors in $B$ and concatenating them following their directions counterclockwise. Equivalently, $P(B)$ is characterized modulo translations by the properties that its inner normal directions are those spanned by the non-zero vectors in $B$ and that for each such inner normal direction, the length of the corresponding edge of $P(B)$ equals the sum of the lengths of the vectors in $B$ in that direction.

The following is main result of this article.

Theorem 1.1. Let $\rho: \mathbb{T}^{1} \rightarrow \mathbb{T}^{2}$ be a non-constant rational map, then

$$
\operatorname{deg}(\rho) \mathrm{N}\left(\overline{\rho\left(\mathbb{T}^{1}\right)}\right)=P\left(\left(\operatorname{ord}_{v}(\rho)\right)_{v \in \mathbb{P}^{1}}\right) .
$$

The notion of Newton polygon can be naturally extended to any effective Weil divisor $Z \in \operatorname{Div}\left(\mathbb{T}^{2}\right)$ : given an equation $E_{Z} \in \mathbb{K}\left[x^{ \pm 1}, y^{ \pm 1}\right]$ for this divisor, the Newton polygon of $Z$ is just defined as the convex hull of the exponents of the monomials in $E_{Z}$. By the definition of the push-forward cycle we have

$$
\rho^{*}\left(\mathbb{T}^{1}\right)=\operatorname{deg}(\rho)\left[\overline{\rho\left(\mathbb{T}^{1}\right)}\right]
$$

and so Theorem 1.1 can be alternatively stated as $\mathrm{N}\left(\rho^{*}\left(\mathbb{T}^{1}\right)\right)=P\left(\left(\operatorname{ord}_{v}(\rho)\right)_{v \in \mathbb{P}^{1}}\right)$.

We illustrate Theorem 1.1 with an example.

Example 1.2. Consider the parametrization $\rho=(f, g)$ with

$$
f(t)=\frac{1}{t(t-1)} \quad, \quad g(t)=\frac{t^{2}-5 t+2}{t} .
$$

We have $\operatorname{ord}_{0}(\rho)=(-1,-1), \operatorname{ord}_{1}(\rho)=(-1,0), \operatorname{ord}_{\infty}(\rho)=(2,-1)$ while for each of the two zeros $v_{1}, v_{2} \in \mathbb{T}^{1}$ of $t^{2}-5 t+2$ we have $\operatorname{ord}_{v_{i}}(\rho)=(0,1)$. Figure 1 below shows these vectors and the polygon $\operatorname{deg}(\rho) \mathrm{N}\left(\overline{\rho\left(\mathbb{T}^{1}\right)}\right)$ obtained by rotating -90 degrees these vectors and adequately concatenating them. Note that the resulting polygon is noncontractible in the sense that it is not a translate of a non-trivial integer multiple of any other lattice polygon. This implies that $\operatorname{deg}(\rho)=1$ and so this is the Newton polygon of the curve $\overline{\rho\left(\mathbb{T}^{1}\right)}$.

We can verify the obtained results by contrasting them with the actual equation of the curve:

$$
E \overline{\rho\left(\mathbb{T}^{1}\right)}(x, y)=1-16 x-4 x^{2}-9 x y-2 x^{2} y-x y^{2} .
$$



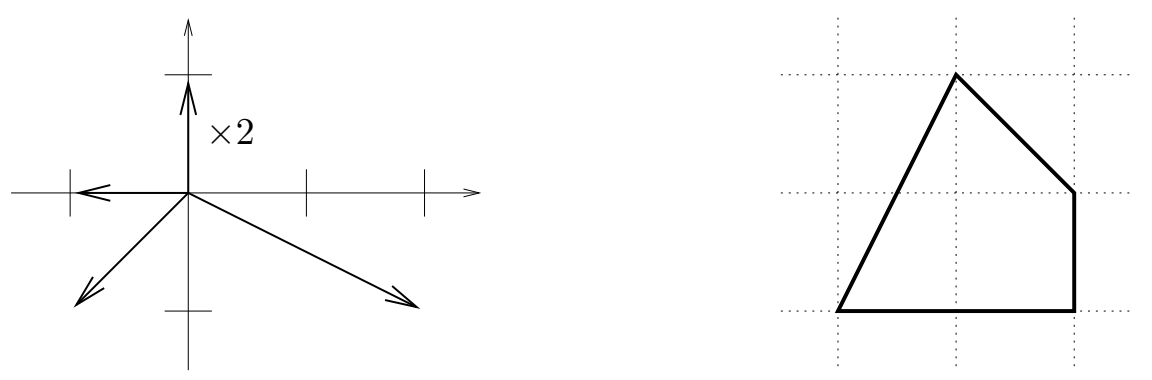

Figure 1. The multiplicities of $\rho$ and the Newton polygon of $\overline{\rho^{*}\left(\mathbb{T}^{1}\right)}$

The computation of $N(C)$ can be done with no need of accessing the zeros and poles of $f$ and $g$. Indeed, suppose that $\mathbb{F}$ is a subfield of $\mathbb{K}$ and $f, g \in \mathbb{F}(t)$. The Newton polygon of $\rho^{*}\left(\mathbb{T}^{1}\right)$ can be obtained from partial factorizations of the form

$$
f(t)=\alpha \prod_{p \in \mathcal{P}} p(t)^{d_{p}} \quad, \quad g(t)=\beta \prod_{p \in \mathcal{P}} p(t)^{e_{p}}
$$

for a finite set $\mathcal{P} \subset \mathbb{F}[t]$ of pairwise coprime polynomials, $d_{p}, e_{p} \in \mathbb{N}$ and $\alpha, \beta \in \mathbb{F}^{\times}$. This principle is already illustrated in the example above and explained for the general case in Lemma 3.3. Such factorizations can be computed with gcd operations only, by applying for instance the algorithm in [PS07b, Lem. 6.2]. It simplifies the computation of the Newton polygon in concrete examples, as it allows to perform it within the field of definition of $\rho$.

The proof of Theorem 1.1 is based on the refinement of the Kušnirenko-Bernštein estimate due to P. Philippon and the second author PS07a, PS07b]. Instead of explicitly dealing with $\mathrm{N}\left(\rho^{*}\left(\mathbb{T}^{1}\right)\right)$, we study the corresponding support function. We show that a certain linear combination of values of this support function can be expressed as an intersection number. We then compute this intersection number by applying the Philippon-Sombra estimate, which turns out to be exact for the present application.

The study of the Newton polytope of a parameterized hypersurface is currently receiving a lot of attention. Part of the motivation is computational: a priori knowledge of the Newton polytope restricts the possible monomials appearing in the implicit equation and thus reduces its computation to Numerical Linear Algebra. Reciprocally, there are algorithms which take as input a multivariate Laurent polynomial and profit from its monomial structure in order to decide more efficiently if the corresponding hypersurface admits a parametrization, and to find one when it has [BS07].

Essentially all of the previous approaches to this problem deal with generic cases, where the monomials of the input parametrization are predetermined and the coefficients are generic. In this form, the problem was first posed in SY94 for Laurent polynomial parametrizations. The first results were obtained by I. Emiris and I. Kotsireas, who computed the Newton polygon and then the implicit equation of some specific parametrizations by determining the extremal monomials of a specialized resultant [EK03, EK05]. This idea is further exploited in [EKP07] to treat other particular cases.

Motivation for this problem comes also from tropical geometry, as the data in the polytope of an effective divisor of $\mathbb{T}^{n}$ is equivalent to the abstract tropical variety associated with the divisor. Based on tropical elimination and intersection theory, 
B. Sturmfels, J. Tevelev and J. Yu have obtained a method for computing the abstract tropical variety associated to a hypersurface parameterized by generic Laurent polynomials in any number of variables [STY07, ST07, SY07]. From another direction, A. Esterov and A. Khovanskiu found an important connection with combinatorics, as they showed that the Newton polytope of the projection of a generic complete intersection is isomorphic to the mixed fiber polytope of the Newton polytopes associated to the input data EKho07.

In this direction, we remark that Theorem 1.1 can easily be rephrased in the language of tropical geometry. Given an effective cycle $Z$ of $\mathbb{T}^{n}$, its abstract tropical variety is a pair $\left(\mathcal{T}_{Z}, m_{Z}\right)$ where $\mathcal{T}_{Z} \subset \mathbb{R}^{n}$ denotes the tropicalization of $Z$ with respect to the trivial valuation of $\mathbb{K}$ and $m_{Z}: \mathcal{T}_{Z}^{0} \rightarrow \mathbb{N} \backslash\{0\}$ is a locally constant function defined on the set of regular points $\mathcal{T}_{Z}^{0}$ of $\mathcal{T}_{Z}$, see [ST07] for the precise definitions. For an effective divisor $Z \in \operatorname{Div}\left(\mathbb{T}^{2}\right)$ this data is equivalent to the Newton polygon: the tropicalization $\mathcal{T}_{Z}$ consists in the union of the inner normal directions of $\mathrm{N}(Z)$, $\mathcal{T}_{Z}^{0}=\mathcal{T}_{Z} \backslash\{(0,0)\}$ and for a point $b \neq(0,0)$ in one of these directions, $m_{Z}(b)$ equals the lattice length of the corresponding edge. Recall that the lattice length $\ell(S)$ of a lattice segment $S \subset \mathbb{R}^{2}$ is the number of points of $\mathbb{Z}^{2}$ on it (including its endpoints) minus 1. Our result can then be rephrased as

$$
\mathcal{T}_{\rho^{*}\left(\mathbb{T}^{1}\right)}=\bigcup_{v \in \mathbb{P}^{1}}\left(\mathbb{R}_{\geq 0}\right) \operatorname{ord}_{v}(\rho),
$$

and $m_{\rho^{*}\left(\mathbb{T}^{1}\right)}(b)=\sum_{v: \operatorname{ord}_{v}(\rho) \in\left(\mathbb{R}_{>0}\right) b} \ell\left(\operatorname{ord}_{v}(\rho)\right)$ for $b \in \mathcal{T}_{\rho^{*}\left(\mathbb{T}^{1}\right)}^{0}$.

Theorem 1.1 above is the first one describing the Newton polygon of a parametrization without any genericity assumption. As an application, we recover the Newton polygon of the generic Laurent polynomial parametrization of dimension 1 obtained in [STY07, ST07] but this time with explicit genericity conditions. Proofs of the following statements can be found in Section 3 ,

Corollary 1.3 (Generic Laurent Polynomials). Let $D \geq d$ and $E \geq e$ and consider the parametrization $\rho=(p, q)$ where

$$
p(t)=\alpha_{d} t^{d}+\cdots+\alpha_{D} t^{D} \quad, \quad q(t)=\beta_{e} t^{e}+\cdots+\beta_{E} t^{E} \quad \in \mathbb{K}\left[t^{ \pm 1}\right]
$$

such that $\alpha_{d}, \alpha_{D}, \beta_{e}, \beta_{E} \neq 0$, then

$$
\operatorname{deg}(\rho) \mathrm{N}\left(\overline{\rho\left(\mathbb{T}^{1}\right)}\right)=P((D-d, 0),(0, E-e),(-D,-E),(d, e))
$$

if and only if $\operatorname{gcd}(p, q)=1$. If moreover the vectors $(D-d, 0),(0, E-e),(d, e)$ are not collinear, then $\operatorname{deg}(\rho)=1$ for generic $p, q$.

The case of parametrizations with generic rational functions with the same denominator is studied in [EKP07, where some heuristics are given concerning its Newton polygon. We solve completely this problem and find that $\mathrm{N}(C)$ has at most five edges.

Corollary 1.4 (Generic Rational Functions with the Same Denominator). Let $D \geq d$, $E \geq e, F \geq 0$ and consider the parametrization $\rho=\left(\frac{p}{r}, \frac{q}{r}\right) \in \mathbb{K}(t)^{2}$ where

$$
p(t)=\alpha_{d} t^{d}+\cdots+\alpha_{D} t^{D} \quad, \quad q(t)=\beta_{e} t^{e}+\cdots+\beta_{E} t^{E} \quad, \quad r(t)=\gamma_{0}+\cdots+\gamma_{F} t^{F}
$$

such that $\alpha_{d}, \alpha_{D}, \beta_{e}, \beta_{E}, \gamma_{0}, \gamma_{F} \neq 0$, then

$$
\operatorname{deg}(\rho) \mathrm{N}\left(\overline{\rho\left(\mathbb{T}^{1}\right)}\right)=P((D-d, 0),(0, E-e),(F-D, F-E),(d, e),(-F,-F))
$$


if and only if $p, q, r$ are pairwise coprime. If moreover the vectors $(D-d, 0),(0, E-$ $e),(d, e),(F, F)$ are not collinear, then $\operatorname{deg}(\rho)=1$ for generic $p, q, r$.

In the case of different denominators, the Newton polygon has at most six edges.

Corollary 1.5 (Generic Rational Functions with Different Denominators). Let $D \geq d$, $E \geq e, F, G \geq 0$, and consider the parametrization $\rho=\left(\frac{p}{r}, \frac{q}{s}\right) \in \mathbb{K}(t)^{2}$ where

$$
p(t)=\alpha_{d} t^{d}+\cdots+\alpha_{D} t^{D} \quad, \quad q(t)=\beta_{e} t^{e}+\cdots+\beta_{E} t^{E} \quad \in \mathbb{K}\left[t^{ \pm 1}\right]
$$

and

$$
r(t)=\gamma_{0}+\cdots+\gamma_{F} t^{F} \quad, \quad s(t)=\delta_{0}+\cdots+\delta_{G} t^{G} \quad \in \mathbb{K}[t]
$$

such that $\alpha_{d}, \alpha_{D}, \beta_{e}, \beta_{E}, \gamma_{0}, \gamma_{F}, \delta_{0}, \delta_{G} \neq 0$, then

$$
\operatorname{deg}(\rho) \mathrm{N}\left(\overline{\rho\left(\mathbb{T}^{1}\right)}\right)=P((D-d, 0),(0, E-e),(F-D, G-E),(d, e),(-F, 0),(0,-G))
$$

if and only if $p, q, r, s$ are pairwise coprime. If moreover the vectors $(D-d, 0),(0, E-$ $e),(d, e),(F, 0),(0, G)$ are not collinear, then $\operatorname{deg}(\rho)=1$ for generic $p, q, r, s$.

Newton polygons arising from the generic cases are very special: they have at most six edges and a particular shape. It is then natural to ask which convex lattice polygons do realize as the Newton polygon of a rational plane curve.

Let $Q \subset \mathbb{R}^{2}$ be an arbitrary lattice convex polygon. Setting $L:=\operatorname{Card}\left(Q \cap \mathbb{Z}^{2}\right)-1$ we identify the space of Laurent polynomials with support contained in $Q$ with $\mathbb{K}^{L+1}$. Consider the set

$$
M_{Q}^{\circ}:=\left\{F \in \mathbb{K}\left[x^{ \pm 1}, y^{ \pm 1}\right]: \mathrm{N}(F)=Q, V(F) \subset \mathbb{T}^{2} \text { is a rational curve }\right\} \subset \mathbb{P}^{L}
$$

and let $M_{Q} \subset \mathbb{P}^{L}$ denote its Zariski closure. The set $M_{Q}^{\circ}$ can be regarded as the space parametrizing divisors of $\mathbb{T}^{2}$ of the form $\delta[C]$ where $C$ is a rational curve, $\delta \geq 1$ and $\mathrm{N}(\delta[C])=Q$.

A convex lattice polygon of $\mathbb{R}^{2}$ is said non-degenerated if it is of dimension 2 or equivalently, if it has positive area. Recall that a variety is unirational if its function field admits a finite extension which is purely transcendental. As a further consequence of Theorem 1.1 we obtain the following result.

Theorem 1.6. Let $Q \subset \mathbb{R}^{2}$ be a non-degenerate convex lattice polygon, then $M_{Q}$ is a unirational variety of dimension $\operatorname{Card}\left(\partial Q \cap \mathbb{Z}^{2}\right)-1$. If moreover $\operatorname{char}(\mathbb{K})=0$, the variety $M_{Q}$ is rational.

Under the assumption that $Q$ is non-degenerate, the polygon has at least three edges and so $\operatorname{dim}\left(M_{Q}\right) \geq 2$. In particular, $M_{Q}^{\circ}$ is non-empty. Moreover, we show in Proposition 4.3 that the generic member of $M_{Q}^{\circ}$ has multiplicity one, and so in this case there always exists a rational plane curve $C$ such that $Q=\mathrm{N}(C)$.

For a lattice segment $S \subset \mathbb{R}^{2}$, we show in Proposition 4.8 that $\operatorname{dim}\left(M_{S}\right)=1$ and that the multiplicity of a general member of $M_{S}^{\circ}$ equals $\ell(S)$. In particular, there exists a rational plane curve $C$ such that $S=\mathrm{N}(C)$ if and only if $S$ does not contain any lattice point except its endpoints.

We also characterize the polygons which can be realized as the Newton polygon of a curve parametrized with polynomials or with Laurent polynomials. 
Theorem 1.7. Let $Q \subset \mathbb{R}^{2}$ be a non-degenerate lattice convex polygon, then $Q=$ $\mathrm{N}\left(\overline{\rho\left(\mathbb{T}^{1}\right)}\right)$ for at least one $\rho \in \mathbb{K}[t]^{2}$ (resp. $\rho \in \mathbb{K}\left[t^{ \pm 1}\right]^{2}$ ) if and only if all but one (resp. one or two) of its inner normal directions lie in $\left(\mathbb{R}_{\geq 0}\right)^{2}$.

This result gives some idea of the discrepancy between general rational curves and curves parameterized by polynomials or Laurent polynomials. For instance, for the polygon in Figure 1 above, the variety $M_{Q}$ is a rational hypersurface of $\mathbb{P}^{5}$ but none of its members is a curve parameterized with polynomials or with Laurent polynomials.

The text is organized as follows: in Section 2 we give a detailed proof of Theorem 1.1. In Section 3 we prove that if $Q$ is a non-degenerate polygon, then the generic parametrization having $Q=\mathrm{N}\left(\rho^{*}\left(\mathbb{T}^{1}\right)\right)$ is birational. We also give an algorithmic criteria in order to compute $\mathrm{N}(C)$ and finally prove Corollaries 1.3, 1.4 and 1.5, In Section 4 we study geometric properties of $M_{Q}$, and give proofs of Theorems 1.6 and 1.7. We conclude by considering the case when $Q$ is a lattice segment.

Acknowledgments. We thank José Ignacio Burgos, Laura Costa, Luis Felipe Tabera and Gerald Welters for helpful comments and conversations. Experiments where carried out with the aid of Maple and M. Franz's package Convex [Fra06.

\section{Proof of Theorem 1.1}

All considered varieties are defined over $\mathbb{K}$, reduced and irreducible. For a family of regular functions $f_{1}, \ldots, f_{s}$ on an algebraic space, we denote by $V\left(f_{1}, \ldots, f_{s}\right)$ the algebraic set they define in this space. A property depending on parameters is said generic if it holds for all points in a dense open subset of the parameter space.

For a rational function $f \in \mathbb{K}(t)^{\times}$we set $f_{\text {num }}, f_{\text {den }} \in \mathbb{K}[t]$ for its numerator and denominator, which are coprime polynomials such that $f=f_{\text {num }} / f_{\text {den }}$; these polynomials are well-defined up to a scalar factor. We denote by

$$
\operatorname{deg}(f):=\operatorname{deg}\left(f_{\text {num }}\right)-\operatorname{deg}\left(f_{\text {den }}\right) \quad, \quad \eta(f):=\max \left\{\operatorname{deg}\left(f_{\text {num }}\right), \operatorname{deg}\left(f_{\text {den }}\right)\right\}
$$

the degree and the height of $f$, respectively.

A convex lattice polygon of $\mathbb{R}^{2}$ is non-degenerated if it is of dimension 2 or equivalently, if it has positive area. We denote by $\mathbb{N}$ and $\mathbb{N}^{\times}$the set of non-negative and positive integers respectively.

With notation as in the introduction, we fix a reduced equation $E_{C} \in \mathbb{K}\left[x^{ \pm 1}, y^{ \pm 1}\right]$ for the plane curve $C=\overline{\rho\left(\mathbb{T}^{1}\right)}$ and we set $\mathrm{N}(C):=\mathrm{N}\left(E_{C}\right) \subset \mathbb{R}^{2}$ for its Newton polygon. A possible way of fixing $E_{C}$ (up to a non-zero scalar) is to suppose that it lies in $\mathbb{K}[x, y]$ and that neither $x$ nor $y$ divide it or equivalently, that $\mathrm{N}(C)$ is contained in the first quadrant and touches both the horizontal and vertical axes. Nevertheless, any other choice will be equally good for what follows.

For $\sigma=\left(\sigma_{1}, \sigma_{2}\right) \in(\mathbb{Z} \backslash\{0\})^{2}$ we set $G(\sigma)$ for the Zariski closure of the set

$$
\left\{(t, x, y): x^{\sigma_{1}}=f(t), y^{\sigma_{2}}=g(t)\right\} \subset \mathbb{T}^{1} \times \mathbb{T}^{2},
$$

which is a pure 1-dimensional algebraic set. Let $\pi: \mathbb{T}^{1} \times \mathbb{T}^{2} \rightarrow \mathbb{T}^{2}$ denote the natural projection onto the second factor and set

$$
C(\sigma):=\overline{\pi(G(\sigma))} \subset \mathbb{T}^{2} .
$$

Note that $G:=G(1,1)$ is the graph of $\rho$, while $C(1,1)=C$. 
We define the degree $\operatorname{deg}(Z)$ of a pure 1-dimensional algebraic set in the torus $\mathbb{T}^{2}$ as its degree with respect to the standard inclusion $\iota: \mathbb{T}^{2} \hookrightarrow \mathbb{P}^{2}$; in other words

$$
\operatorname{deg}(Z)=\operatorname{Card}(\iota(Z) \cap L)
$$

for a generic line $L \subset \mathbb{P}^{2}$.

Given a Laurent polynomial $F \in \mathbb{K}\left[x^{ \pm 1}, y^{ \pm 1}\right]$ we denote by $\operatorname{deg}_{x}(F)\left(r e s p \cdot \operatorname{deg}_{y}(F)\right)$ its degree in the variable $x$ (resp. $y$ ), and $\operatorname{deg}(F)$ its total degree in $x$ and $y$. Its homogenization with respect to $\iota$ can then be written as $F^{\mathrm{h}}(w, x, y)=w^{\delta} F(x / w, y / w)$ with

$$
\delta=\operatorname{deg}(F(x, y))+\operatorname{deg}_{x}\left(F\left(x^{-1}, y\right)\right)+\operatorname{deg}_{y}\left(F\left(x, y^{-1}\right)\right) .
$$

Hence in case $F$ is reduced, we have

$$
\operatorname{deg}(V(F))=\operatorname{deg}(F(x, y))+\operatorname{deg}_{x}\left(F\left(x^{-1}, y\right)\right)+\operatorname{deg}_{y}\left(F\left(x, y^{-1}\right)\right) .
$$

The support function of a convex set $Q \subset \mathbb{R}^{2}$ is defined as

$$
h(Q ; \cdot): \mathbb{R}^{2} \rightarrow \mathbb{R} \quad, \quad w \mapsto \max \{\langle w, u\rangle, u \in Q\} .
$$

This is a piecewise affine convex function, which completely characterizes $Q$ as the set of points $u \in \mathbb{R}^{2}$ such that $\langle w, u\rangle \leq h(Q ; w)$ for all $w \in \mathbb{R}^{2}$. Note that for $F \in \mathbb{K}\left[x^{ \pm 1}, y^{ \pm 1}\right]$ such that $\mathrm{N}(F)=Q$ and $\sigma=\left(\sigma_{1}, \sigma_{2}\right) \in \mathbb{Z}$ we have $\operatorname{deg}\left(F\left(x^{\sigma_{1}}, y^{\sigma_{2}}\right)\right)=$ $h(Q ; \sigma)$. This fact will be used in the sequel.

The following result expresses a linear combination of support functions of $\mathrm{N}(C)$ as an intersection number. It can be regarded as some kind of extension of the "Perron's theorem" in [Jel05, Thm. 3.3].

Proposition 2.1. With notation as above, set $p:=\operatorname{char}(\mathbb{K})$ and let $\sigma_{1}, \sigma_{2} \in \mathbb{Z} \backslash p \mathbb{Z}$, then $\operatorname{deg}\left(\left.\pi\right|_{G(\sigma)}\right)=\operatorname{deg}(\rho)$ and

$$
h\left(\mathrm{~N}(C) ;\left(\sigma_{1}, \sigma_{2}\right)\right)+h\left(\mathrm{~N}(C) ;\left(-\sigma_{1}, 0\right)\right)+h\left(\mathrm{~N}(C) ;\left(0,-\sigma_{2}\right)\right)=\operatorname{deg}\left(C\left(\sigma_{1}, \sigma_{2}\right)\right) .
$$

Proof. Let $\pi: \mathbb{T}^{1} \times \mathbb{T}^{2} \rightarrow \mathbb{T}^{2}$ denote the natural projection onto the second factor and $\chi: \mathbb{T}^{2} \rightarrow \mathbb{T}^{2}$ the monomial map $(x, y) \mapsto\left(x^{\sigma_{1}}, y^{\sigma_{2}}\right)$. Set $\sigma:=\left(\sigma_{1}, \sigma_{2}\right)$ and consider the commutative diagram

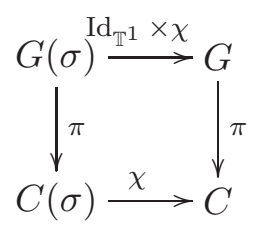

The horizontal arrows are finite coverings of the same degree $\left|\sigma_{1} \sigma_{2}\right|$. On the other hand

$\operatorname{deg}(\chi) \operatorname{deg}\left(\left.\pi\right|_{G(\sigma)}\right)=\operatorname{deg}\left(\left.\chi \circ \pi\right|_{G(\sigma)}\right)=\operatorname{deg}\left(\left.\pi\right|_{G} \circ\left(\operatorname{Id}_{\mathbb{T}^{1}} \times \chi\right)\right)=\operatorname{deg}\left(\left.\pi\right|_{G}\right) \operatorname{deg}\left(\operatorname{Id}_{\mathbb{T}^{1}} \times \chi\right)$, which implies the first part of the proposition: $\operatorname{deg}\left(\left.\pi\right|_{G(\sigma)}\right)=\operatorname{deg}\left(\left.\pi\right|_{G}\right)=\operatorname{deg}(\rho)$.

For the second part, we claim that $\chi^{*}\left(E_{C}\right)=E_{C}\left(x^{\sigma_{1}}, y^{\sigma_{2}}\right)$ is a reduced equation for $C(\sigma)$. We recall that a Laurent polynomial $F \in \mathbb{K}\left[x^{ \pm 1}, y^{ \pm 1}\right]$ is reduced if and only if its gradient $\nabla(F)=(\partial F / \partial x, \partial F / \partial y)$ does not vanish on any of the components of $V(F)$, see for instance [Jel05, Lem. 3.1]. This condition automatically holds for $\chi^{*}\left(E_{C}\right)$, because by construction it holds for $E_{C}$, and $\sigma_{1}, \sigma_{2} \notin p \mathbb{Z}$. Hence $\chi^{*}\left(E_{C}\right)$ is reduced. On the other hand, it is clear that $C(\sigma)$ coincides with the zero set of $\chi^{*}\left(E_{C}\right)$, which proves the claim. 
Identity (2.1) implies then

$$
\begin{aligned}
\operatorname{deg}(C(\sigma)) & =\operatorname{deg}\left(\chi^{*}\left(E_{C}\right)(x, y)\right)+\operatorname{deg}_{x}\left(\chi^{*}\left(E_{C}\right)\left(x^{-1}, y\right)\right)+\operatorname{deg}_{y}\left(\chi^{*}\left(E_{C}\right)\left(x, y^{-1}\right)\right) \\
& =\operatorname{deg}\left(E\left(x^{\sigma_{1}}, y^{\sigma_{2}}\right)\right)+\operatorname{deg}_{x}\left(E\left(x^{-\sigma_{1}}, y^{\sigma_{2}}\right)\right)+\operatorname{deg}_{y}\left(E\left(x^{\sigma_{1}}, y^{-\sigma_{2}}\right)\right) \\
& =h\left(\mathrm{~N}(C) ;\left(\sigma_{1}, \sigma_{2}\right)\right)+h\left(\mathrm{~N}(C) ;\left(-\sigma_{1}, 0\right)\right)+h\left(\mathrm{~N}(C) ;\left(0,-\sigma_{2}\right)\right)
\end{aligned}
$$

Note that the algebraic set $G(\sigma)$ can be written as

$$
G(\sigma)=V\left(f_{\operatorname{den}}(t) x^{\sigma_{1}}-f_{\text {num }}(t), g_{\operatorname{den}}(t) y^{\sigma_{2}}-g_{\text {num }}(t)\right) .
$$

For a generic line $L \subset \mathbb{P}^{2}$, we have that $G(\sigma) \cap\left(\mathbb{T}^{1} \times \iota^{-1}(L)\right)=\pi^{-1}(C(\sigma) \cap L)$ and so

$$
\operatorname{Card}\left(G(\sigma) \cap\left(\mathbb{T}^{1} \times \iota^{-1}(L)\right)\right)=\operatorname{deg}\left(\left.\pi\right|_{G(\sigma)}\right) \operatorname{deg}(C(\sigma)) .
$$

Hence Proposition 2.1 implies that for $\sigma_{1}, \sigma_{2} \in \mathbb{Z} \backslash p \mathbb{Z}$, the quantity

$$
\operatorname{deg}(\rho)\left(h\left(\mathrm{~N}(C) ;\left(\sigma_{1}, \sigma_{2}\right)\right)+h\left(\mathrm{~N}(C) ;\left(-\sigma_{1}, 0\right)\right)+h\left(\mathrm{~N}(C) ;\left(0,-\sigma_{2}\right)\right)\right)
$$

equals the number of solutions in $\mathbb{T}^{1} \times \mathbb{T}^{2}$ of the system of equations

$$
\begin{array}{r}
\ell_{0}+\ell_{1} x+\ell_{2} y=0 \\
f_{\text {num }}(t)-f_{\text {den }}(t) x^{\sigma_{1}}=0 \\
g_{\text {num }}(t)-g_{\text {den }}(t) y^{\sigma_{2}}=0
\end{array}
$$

for generic $\ell_{0}, \ell_{1}, \ell_{2} \in \mathbb{K}$.

The number of solutions of this system can be expressed in combinatorial terms thanks to a result of Philippon and the second author PS07a, PS07b. We introduce some combinatorial invariants in order to explain it better. Restricting to our setting, let

$$
H(t, x, y)=\sum_{j=0}^{M} \alpha_{j}(t) x^{a_{j}} y^{b_{j}} \in \mathbb{K}(t)\left[x^{ \pm 1}, y^{ \pm 1}\right]
$$

be a non-zero Laurent polynomial in the variables $x, y$ with coefficients rational functions in the variable $t$. For each $v \in \mathbb{P}^{1}$ consider the $v$-adic Newton polytope of $H$

$$
\mathrm{N}_{v}(H):=\operatorname{Conv}\left(\left(a_{0}, b_{0},-\operatorname{ord}_{v}\left(\alpha_{0}\right)\right), \ldots,\left(a_{M}, b_{M},-\operatorname{ord}_{v}\left(\alpha_{M}\right)\right)\right) \subset \mathbb{R}^{3} .
$$

This polytope sits above the usual Newton polygon

$$
\mathrm{N}(H):=\operatorname{Conv}\left(\left(a_{0}, b_{0}\right), \ldots,\left(a_{M}, b_{M}\right)\right) \subset \mathbb{R}^{2}
$$

via the natural projection $\mathbb{R}^{3} \rightarrow \mathbb{R}^{2}$ which forgets the last coordinate. Consider the roof function of $H$ at $v$ defined as

$$
\vartheta_{v}(H): \mathrm{N}(H) \rightarrow \mathbb{R} \quad, \quad(x, y) \mapsto \max \left\{z \in \mathbb{R}:(x, y, z) \in \mathrm{N}_{v}(H)\right\}
$$

that is, the concave and piecewise affine function parameterizing the upper envelope of $\mathrm{N}_{v}(H)$ above $\mathrm{N}(H)$.

For the polynomials in the system (2.4), the respective Newton polygons are

$$
\begin{aligned}
& P_{0}:=\mathrm{N}\left(\ell_{0}+\ell_{1} x+\ell_{2}\right)=\operatorname{Conv}((0,0),(1,0),(0,1)), \\
& P_{1}:=\mathrm{N}\left(f_{\text {num }}(t)-f_{\text {den }}(t) x^{\sigma_{1}}\right)=\operatorname{Conv}\left((0,0),\left(\sigma_{1}, 0\right)\right), \\
& P_{2}:=\mathrm{N}\left(g_{\text {num }}(t)-g_{\text {den }}(t) y^{\sigma_{2}}\right)=\operatorname{Conv}\left((0,0),\left(0, \sigma_{2}\right)\right),
\end{aligned}
$$


so that $P_{0}$ is the standard triangle in $\mathbb{R}^{2}$ while $P_{1}$ and $P_{2}$ are segments. For $v \in \mathbb{P}^{1}$ and $i=0,1,2$ we denote $P_{i, v} \subset \mathbb{R}^{3}$ and $\vartheta_{i, v}: P_{i} \rightarrow \mathbb{R}$ the $v$-adic Newton polytope and corresponding roof function for the polynomials $\ell_{0}+\ell_{1} x+\ell_{2} y, f_{\text {den }}(t) x^{\sigma_{1}}-f_{\text {num }}(t)$ and $g_{\text {den }}(t) y^{\sigma_{2}}-g_{\text {num }}(t)$, respectively. Computing them explicitly, we get $\vartheta_{0, v} \equiv 0$, while for $i=1,2$ we have that $\vartheta_{i, v}$ is the affine function on $P_{i}$ such that

$$
\vartheta_{1, v}(0,0)=-\operatorname{ord}_{v}\left(f_{\text {num }}\right) \quad, \quad \vartheta_{1, v}\left(\sigma_{1}, 0\right)=-\operatorname{ord}_{v}\left(f_{\text {den }}\right)
$$

and

$$
\vartheta_{2, v}(0,0)=-\operatorname{ord}_{v}\left(g_{\text {num }}\right) \quad, \quad \vartheta_{2, v}\left(0, \sigma_{2}\right)=-\operatorname{ord}_{v}\left(g_{\text {den }}\right) .
$$

Note that $\vartheta_{i, v} \leq 0$ for $v \neq \infty$ while $\vartheta_{i, v} \geq 0$ for $v=\infty$. The figure below shows the graph of these roof functions.

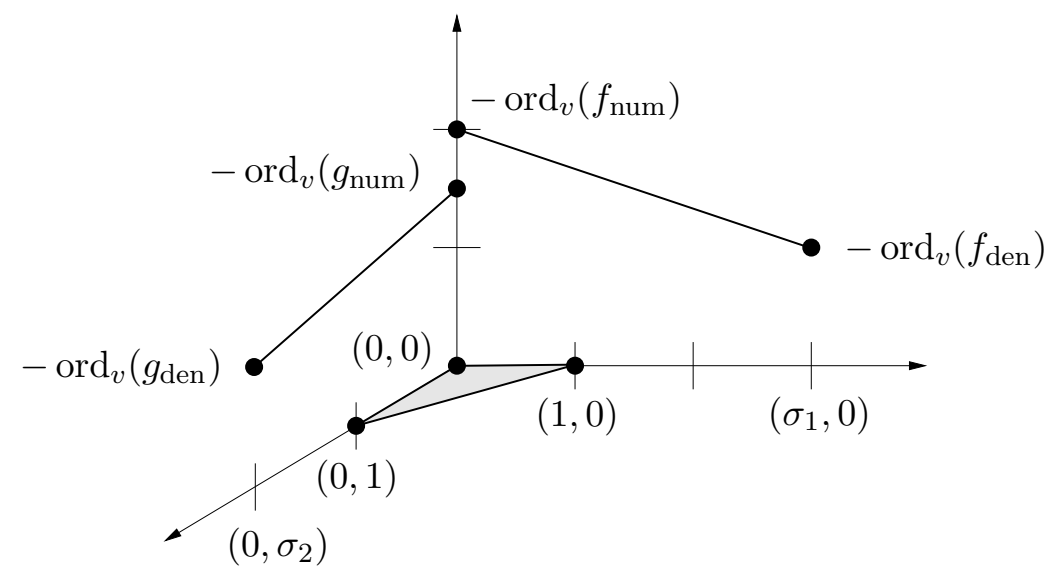

Figure 2. The $v$-adic Newton polytopes for the system (2.4)

As the polynomials in (2.4) are primitive we can apply [PS07b, Thm. 1.2], which shows that the number of solutions is bounded above by the quantity

$$
\sum_{v \in \mathbb{P}^{1}} \mathrm{MI}_{2}\left(\vartheta_{0, v}, \vartheta_{1, v}, \vartheta_{2, v}\right) \text {. }
$$

The mixed integral MI in this expression is the natural extension to concave functions of the mixed volume of convex bodies. As such, it satisfies analogous properties. We refer to [PS04, $\S \mathrm{IV}]$ and $[\mathrm{PS} 07 \mathrm{~b}, \S 8]$ for its definition and basic properties.

The estimate (2.5) is exact in this case because none of the relevant initial systems has a root. Initial systems in this context can be interpreted as the restriction of the input system to the face of the upper envelope of the $v$-adic Newton polytopes $P_{i, v}$ 's corresponding to $\tau$. We will explain them briefly here, but refer the reader interested in more details to PS07b, $\S 6]$.

Let $H \in \mathbb{K}(t)\left[x^{ \pm 1}, y^{ \pm 1}\right]$ and $\tau \in \mathbb{R}^{2}$, then for $v \in \mathbb{P}^{1} \backslash\{\infty\}$ the $\tau$-initial part of $H$ at $v$ is defined as the Laurent polynomial $\operatorname{init}_{v, \tau}(H) \in \mathbb{K}\left[x^{ \pm 1}, y^{ \pm 1}\right]$ such that

$$
H\left(t, t^{\tau_{1}} x, t^{\tau_{2}} y\right)=(t-v)^{c}\left(\operatorname{init}_{v, \tau}(H)(x, y)+o(1)\right)
$$

for a $c \in \mathbb{Z}$ and $o(1)$ going to 0 as $t \rightarrow v$, while the $\tau$-initial part of $H$ at $\infty$ is just defined as the $\tau$-initial part of $H\left(t^{-1}, x, y\right)$ at 0 .

By [PS07b, Prop. 1.4], if for all $v \in \mathbb{P}^{1} \backslash\{\infty\}$ and $\tau \neq(0,0)$, and for $v=\infty$ and all $\tau \in \mathbb{R}^{2}$, the system of equations

$$
\operatorname{init}_{v, \tau}\left(\ell_{0}+\ell_{1} x+\ell_{2}\right)=\operatorname{init}_{v, \tau}\left(f_{\text {num }}(t)-f_{\text {den }}(t) x^{\sigma_{1}}\right)=\operatorname{init}_{v, \tau}\left(g_{\text {num }}(t)-g_{\text {den }}(t) y^{\sigma_{2}}\right)=0
$$


has no solution in $\mathbb{T}^{2}$, then the estimate (2.5) counts exactly the number of roots of the system in $\mathbb{A}^{1} \times \mathbb{T}^{2}$.

In our setting, at least one of the initial polynomials

$$
\operatorname{init}_{v, \tau}\left(f_{\text {num }}(t)-f_{\text {den }}(t) x^{\sigma_{1}}\right) \quad, \quad \operatorname{init}_{v, \tau}\left(g_{\text {num }}(t)-g_{\text {den }}(t) y^{\sigma_{2}}\right)
$$

reduces to a monomial (and hence the initial system has no solution in $\mathbb{T}^{2}$ ) unless

$$
\tau=\tau(v):=\left(-\frac{\operatorname{ord}_{v}(f)}{\sigma_{1}},-\frac{\operatorname{ord}_{v}(g)}{\sigma_{2}}\right) .
$$

We have that $\tau(v)=(0,0)$ for all but a finite number of $v$ 's and by the stated equality criterion these $v$ 's need not be considered. On the other hand, the finite number of $v$ 's such that $\tau(v) \neq(0,0)$ produces a finite number of solutions for the initial system (2.6), all of which are avoided by the linear form $\ell_{0}+\ell_{1} x+\ell_{2} y$, as this last one is supposed generic.

Hence none of the relevant initial systems have solutions and so (2.5) is an equality, as announced. We conclude that for $\sigma_{1}, \sigma_{2} \in \mathbb{Z} \backslash p \mathbb{Z}$ the degree of the curve $C(\sigma)$ can be expressed as

$$
\operatorname{deg}(C(\sigma))=\frac{1}{\operatorname{deg}(\rho)} \sum_{v \in \mathbb{P}^{1}} \operatorname{MI}_{2}\left(\vartheta_{0, v}, \vartheta_{1, v}, \vartheta_{2, v}\right) .
$$

We extend this formula to $\sigma \in\left(\mathbb{R}^{\times}\right)^{2}$ and make it explicit by computing the relevant mixed integrals.

Proposition 2.2. With notation as before, let $\sigma \in\left(\mathbb{R}^{\times}\right)^{2}$, then

$$
\begin{aligned}
& h\left(\mathrm{~N}(C) ;\left(\sigma_{1}, \sigma_{2}\right)\right)+h\left(\mathrm{~N}(C) ;\left(-\sigma_{1}, 0\right)\right)+h\left(\mathrm{~N}(C) ;\left(0,-\sigma_{2}\right)\right) \\
& \quad=\frac{1}{\operatorname{deg}(\rho)} \sum_{v \in \mathbb{P}^{1}} \max \left\{0,-\operatorname{ord}_{v}(g) \operatorname{sign}\left(\sigma_{1} \sigma_{2}\right) \sigma_{1},-\operatorname{ord}_{v}(f) \operatorname{sign}\left(\sigma_{1} \sigma_{2}\right) \sigma_{2}\right\} .
\end{aligned}
$$

Proof. Suppose first $\sigma_{1}, \sigma_{2} \in \mathbb{Z} \backslash p \mathbb{Z}$. We compute the mixed integrals in Formula (2.5) by applying the decomposition formula PS07b, Formula (8.6)]: with notation as before, for each $v \in \mathbb{P}^{1}$ we have

$$
\begin{aligned}
\operatorname{MI}_{2}\left(\vartheta_{0, v}, \vartheta_{1, v}, \vartheta_{2, v}\right)= & \sum_{u \in S^{1}} h\left(P_{0} ; u\right) \operatorname{MI}_{1}\left(\left.\vartheta_{1, v}\right|_{P_{1}^{u}},\left.\vartheta_{2, v}\right|_{P_{2}^{u}}\right) \\
& +\sum_{y \in S_{+}^{2}} h\left(P_{0, v} ; y\right) \operatorname{MV}_{2}\left(P_{1, v}^{y}, P_{2, v}^{y}\right) .
\end{aligned}
$$

Here $S^{1} \subset \mathbb{R}^{2}$ denotes the unit circle and $S_{+}^{2} \subset \mathbb{R}^{2}$ denotes the set of points in the sphere $S^{2}$ whose last coordinate is positive, while $P_{i}^{u}\left(\right.$ resp. $\left.P_{i, v}^{y}\right)$ stands for the face (resp. the slope) in the direction $u$ (resp. y) of $P_{i}$ (resp. $\left.P_{i, v}\right)$.

For each $v \in \mathbb{P}^{1}$, set $I_{v}$ for the first sum. We have

$$
I_{v}=h\left(P_{0} ;(1,0)\right) \operatorname{MI}_{1}\left(\left.\vartheta_{1, v}\right|_{P_{1}^{(1,0)}}, \vartheta_{2, v}\right)+h\left(P_{0} ;(0,1)\right) \operatorname{MI}_{1}\left(\vartheta_{1, v},\left.\vartheta_{2, v}\right|_{P_{2}^{(0,1)}}\right) .
$$

It turns out that $P_{1}^{(1,0)}$ must be equal to one of the points $\left(\sigma_{1}, 0\right)$ or $(0,0)$ depending on the sign of $\sigma_{1}$. Similarly, $P_{2}^{(0,1)}$ must be equal to either $\left(0, \sigma_{2}\right)$ or $(0,0)$ depending on the sign of $\sigma_{2}$. By [PS07b, Formula (8.3)]

$$
I_{v}=\left|\sigma_{1}\right| \vartheta_{1, v}\left(P_{1}^{(1,0)}\right)+\left|\sigma_{2}\right| \vartheta_{2, v}\left(P_{2}^{(0,1)}\right)
$$


and so $\sum_{v} I_{v}=0$, because $\vartheta_{i, v}\left(P_{i}^{e_{i}}\right)$ equals the order of vanishing at $v$ of a rational function $q \in \mathbb{K}(t)^{\times}$, and the identity $\sum_{v \in \mathbb{P}^{1}} \operatorname{ord}_{v}(q)=0$ always holds.

Set $J_{v}$ for the second sum, which consists in the only term corresponding to the vector $y \in S_{+}^{2}$ such that $P_{1, v}^{y}=P_{1, v}$ and $P_{2, v}^{y}=P_{2, v}$. Setting $V:=\left(\sigma_{1}, 0, \operatorname{ord}_{v}(f)\right)$ and $W:=\left(0, \sigma_{2}, \operatorname{ord}_{v}(g)\right)$, their exterior product equals

$$
V \times W=\left(-\operatorname{ord}_{v}(f) \sigma_{2},-\operatorname{ord}_{v}(g) \sigma_{1}, \sigma_{1} \sigma_{2}\right),
$$

and so the only relevant $y$ is

$$
y=\frac{\operatorname{sign}\left(\sigma_{1} \sigma_{2}\right)}{\|V \times W\|} V \times W .
$$

We have then $\operatorname{MV}_{2}\left(P_{1, v}^{y}, P_{2, v}^{y}\right)=\|V \times W\|_{2}$, hence

$$
\begin{aligned}
J_{v} & =h\left(P_{0} ; y\right) \operatorname{MV}_{2}\left(P_{1, v}^{y}, P_{2, v}^{y}\right) \\
& =\max \left\{0,-\operatorname{ord}_{v}(f) \operatorname{sign}\left(\sigma_{1} \sigma_{2}\right) \sigma_{2},-\operatorname{ord}_{v}(g) \operatorname{sign}\left(\sigma_{1} \sigma_{2}\right) \sigma_{1}\right\} .
\end{aligned}
$$

Finally, note that both sides of the identity are continuous and homogeneous with respect to homotheties, which implies the general case $\sigma_{1}, \sigma_{2} \in \mathbb{R}^{\times}$.

For each $v \in \mathbb{P}^{1}$ consider a rectangular triangle $R_{v}$ defined as

$$
R_{v}:=\operatorname{Conv}\left((0,0),\left(-\operatorname{ord}_{v}(g), 0\right),\left(0,-\operatorname{ord}_{v}(f)\right)\right)
$$

if $\operatorname{ord}_{v}(f) \operatorname{ord}_{v}(g) \geq 0$ and

$$
R_{v}:=\operatorname{Conv}\left(\left(-\operatorname{ord}_{v}(g),-\operatorname{ord}_{v}(f)\right),\left(-\operatorname{ord}_{v}(g), 0\right),\left(0,-\operatorname{ord}_{v}(f)\right)\right)
$$

if $\operatorname{ord}_{v}(f) \operatorname{ord}_{v}(g) \leq 0$. Both definitions coincide in case $\operatorname{ord}_{v}(f) \operatorname{ord}_{v}(g)=0$. Note that $R_{v}=(0,0)$ for all but a finite number of $v$ 's. The following result gives an explicit expression for the Minkowski difference of $\mathrm{N}(C)$ with the minimal rectangle containing it.

Proposition 2.3. With the above notation, for $i=1,2$ let $\pi_{i}: \mathbb{R}^{2} \rightarrow \mathbb{R}$ denote the projection to the $i$-th factor, then

$$
\mathrm{N}(C)-\pi_{1}(\mathrm{~N}(C)) \times \pi_{2}(\mathrm{~N}(C))=\frac{1}{\operatorname{deg}(\rho)} \sum_{v \in \mathbb{P}^{1}} R_{v} .
$$

Proof. Set for short

$$
D:=\operatorname{deg}(\rho)\left(\mathrm{N}(C)-\pi_{1}(\mathrm{~N}(C)) \times \pi_{2}(\mathrm{~N}(C))\right) \subset \mathbb{R}^{2} .
$$

It suffices to show that the support function of both $D$ and $\sum_{v} R_{v}$ coincide for all $\sigma \in\left(\mathbb{R}^{\times}\right)^{2}$.

In case $\sigma_{1} \sigma_{2}>0$ set $T_{v}:=\operatorname{Conv}\left((0,0),\left(-\operatorname{ord}_{v}(g), 0\right),\left(0,-\operatorname{ord}_{v}(f)\right)\right)$, so that Proposition 2.2 implies that

$$
h(D ; \sigma)=\sum_{v} h\left(T_{v} ; \sigma\right) .
$$

When $\operatorname{ord}_{v}(f) \operatorname{ord}_{v}(g) \geq 0$ we have that $T_{v}=R_{v}$, while if $\operatorname{ord}_{v}(f) \operatorname{ord}_{v}(g) \leq 0$, the maximum of the scalar product of $\sigma$ over the rectangle $R_{v} \cup T_{v}$ is attained either at the point $\left(-\operatorname{ord}_{v}(g), 0\right)$ or at $\left(0,-\operatorname{ord}_{v}(f)\right)$, hence also in this case $h\left(T_{v} ; \sigma\right)=h\left(R_{v} ; \sigma\right)$. Thus

$$
h(D ; \sigma)=\sum_{v} h\left(R_{v} ; \sigma\right) .
$$


In case $\sigma_{1} \sigma_{2}<0$, we use the identities

$$
\sum_{v} \operatorname{ord}_{v}(f)=\sum_{v} \operatorname{ord}_{v}(g)=0
$$

For $v \in \mathbb{P}^{1}$ set

$$
U_{v}:=\operatorname{Conv}\left(\left(-\operatorname{ord}_{v}(g),-\operatorname{ord}_{v}(f)\right),\left(-\operatorname{ord}_{v}(g), 0\right),\left(0,-\operatorname{ord}_{v}(f)\right)\right),
$$

Proposition 2.2 then implies that

$$
\begin{aligned}
h(D ; \sigma)= & \sum_{v} \max \left\{\langle\sigma,(0,0)\rangle,\left\langle\sigma,\left(\operatorname{ord}_{v}(g), 0\right)\right\rangle,\left\langle\sigma,\left(0, \operatorname{ord}_{v}(f)\right)\right\rangle\right\} \\
& -\sum_{v}\left\langle\sigma,\left(\operatorname{ord}_{v}(g), \operatorname{ord}_{v}(f)\right)\right\rangle \\
= & \sum_{v} \max \left\{\left\langle\sigma,\left(-\operatorname{ord}_{v}(g),-\operatorname{ord}_{v}(f)\right)\right\rangle,\left\langle\sigma,\left(-\operatorname{ord}_{v}(g), 0\right)\right\rangle,\left\langle\sigma,\left(0,-\operatorname{ord}_{v}(f)\right)\right\rangle\right\} \\
= & \sum_{v} h\left(U_{v} ; \sigma\right) .
\end{aligned}
$$

The rest of the argument is as in the preceding case: when $\operatorname{ord}_{v}(f) \operatorname{ord}_{v}(g) \leq 0$ we have that $U_{v}=R_{v}$, while if $\operatorname{ord}_{v}(f) \operatorname{ord}_{v}(g) \geq 0$, the maximum of the scalar product of $\sigma$ over the rectangle $R_{v} \cup U_{v}$ is attained either at the point $\left(-\operatorname{ord}_{v}(g), 0\right)$ or at $\left(0,-\operatorname{ord}_{v}(f)\right)$, hence also in this case $h\left(U_{v} ; \sigma\right)=h\left(R_{v} ; \sigma\right)$. We conclude that for all $\sigma \in\left(\mathbb{R}^{\times}\right)^{2}$

$$
h(D ; \sigma)=\sum_{v} h\left(R_{v} ; \sigma\right),
$$

while on the other hand $h\left(\sum_{v} R_{v} ; \sigma\right)=\sum_{v} h\left(R_{v} ; \sigma\right)$ by [Sch93, Thm. 1.7.5, p. 41], which concludes the proof.

Example 2.4. Consider the rational functions

$$
f(t):=\frac{t(t-2)}{(t-1)^{2}} \quad, \quad g(t):=\frac{t(t-1)^{2}}{(t-2)^{3}} .
$$

We have $\operatorname{ord}_{0}(f, g)=(1,1), \operatorname{ord}_{1}(f, g)=(-2,2), \operatorname{ord}_{2}(f, g)=(1,-3)$ and $\operatorname{ord}_{v}(f, g)=$ $(0,0)$ for $v \neq 0,1,2$. Figure 3 below illustrates the sum of the corresponding rectangular triangles. The dashed lines indicate the Newton polygon of the implicit equation for this example.

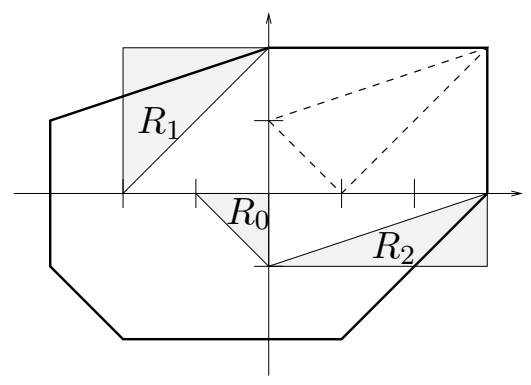

Figure 3. The Minkowski sum of the $R_{v}$ 's 
Now we would like to compute the minimal rectangle containing $\mathrm{N}(C)$. For simplicity, we fix $E_{C}$ up to a non-zero scalar, by supposing it lies in $\mathbb{K}[x, y]$ and that neither $x$ nor $y$ divide it.

Lemma 2.5. With the above notation, we have

$$
\operatorname{deg}(\rho) \operatorname{deg}_{x}\left(E_{C}\right)=\eta(g) \quad, \quad \operatorname{deg}(\rho) \operatorname{deg}_{y}\left(E_{C}\right)=\eta(f) .
$$

Proof. With the given choice of $E_{C}$, we have that

$$
\operatorname{deg}_{x}\left(E_{C}\right)=h(\mathrm{~N}(C) ;(1,0))+h(\mathrm{~N}(C) ;(-1,0))+h(\mathrm{~N}(C) ;(0,0)) .
$$

By Proposition 2.2 applied to $\sigma_{1}=1$ and $\sigma_{2} \rightarrow 0^{+}$we obtain

$$
\begin{aligned}
\operatorname{deg}(\rho) \operatorname{deg}_{x}\left(E_{C}\right) & =\sum_{v \in \mathbb{P}^{1}} \max \left\{0,-\operatorname{ord}_{v}(g)\right\} \\
& =\max \left\{0,-\operatorname{ord}_{\infty}(g)\right\}+\sum_{v \in \mathbb{A}^{1}} \max \left\{0,-\operatorname{ord}_{v}(g)\right\} \\
& =\max \left\{0, \operatorname{deg}\left(g_{\text {num }}\right)-\operatorname{deg}\left(g_{\text {den }}\right)\right\}+\operatorname{deg}\left(g_{\text {den }}\right) \\
& =\max \left\{\operatorname{deg}\left(g_{\text {num }}\right), \operatorname{deg}\left(g_{\text {den }}\right)\right\}=\eta(g) .
\end{aligned}
$$

The expression for $\operatorname{deg}_{y}\left(E_{C}\right)$ follows similarly.

This lemma is equivalent to

$$
\pi_{1}(\mathrm{~N}(C))=\frac{1}{\operatorname{deg}(\rho)} \operatorname{Conv}(0, \eta(g)) \quad, \quad \pi_{2}(\mathrm{~N}(C))=\frac{1}{\operatorname{deg}(\rho)} \operatorname{Conv}(0, \eta(f)) .
$$

Now that we know the size of this minimal rectangle, we are in position to compute $\mathrm{N}(C)$ by "extracting" it from the expression in Proposition 2.3 .

Proof of theorem 1.1. As a first step, we determine the edge structure of the polygon

$$
R:=\sum_{v \in \mathbb{P}^{1}} R_{v}
$$

Let $v \in \mathbb{P}^{1}$. In case $\operatorname{ord}_{v}(f) \operatorname{ord}_{v}(g)>0$ we have that

$$
\left\langle\operatorname{ord}_{v}(\rho),\left(-\operatorname{ord}_{v}(g), 0\right)\right\rangle=\left\langle\operatorname{ord}_{v}(\rho),\left(0,-\operatorname{ord}_{v}(f)\right)\right\rangle=-\operatorname{ord}_{v}(f) \operatorname{ord}_{v}(g)<0
$$

while $\left\langle\operatorname{ord}_{v}(\rho),(0,0)\right\rangle=0$. Hence for each $v$, the rectangular triangle $R_{v}$ contributes to $R$ with

- one edge of length $\left\|\operatorname{ord}_{v}(\rho)\right\|$ and inner normal $\operatorname{ord}_{v}(\rho)$;

- one vertical edge of length $\left|\operatorname{ord}_{v}(g)\right|$ and inner normal $\left(\operatorname{sign}\left(-\operatorname{ord}_{v}(f)\right), 0\right)$;

- one horizontal edge of length $\left|\operatorname{ord}_{v}(f)\right|$ and inner normal $\left(0, \operatorname{sign}\left(-\operatorname{ord}_{v}(g)\right)\right)$.

In case $\operatorname{ord}_{v}(f) \operatorname{ord}_{v}(g)<0$, with a similar computation we can verify that $R_{v}$ contributes to $R$ with

- one edge of length $\left\|\operatorname{ord}_{v}(\rho)\right\|$ and inner normal $\operatorname{ord}_{v}(\rho)$;

- one vertical edge of length $\left|\operatorname{ord}_{v}(g)\right|$ and inner normal $\left(\operatorname{sign}\left(-\operatorname{ord}_{v}(f)\right), 0\right)$;

- one horizontal edge of length $\left|\operatorname{ord}_{v}(f)\right|$ and inner normal $\left(0, \operatorname{sign}\left(-\operatorname{ord}_{v}(g)\right)\right)$; 
exactly as in the first case.

If $\operatorname{ord}_{v}(f)=0$ and $\operatorname{ord}_{v}(g) \neq 0$, the triangle $R_{v}$ contributes to $R$ with two horizontal edges of length $\left|\operatorname{ord}_{v}(g)\right|$, with inner normal $(0,1)$ and $(0,-1)$ respectively. Similarly, in case $\operatorname{ord}_{v}(g)=0$ and $\operatorname{ord}_{v}(f) \neq 0$, the triangle $R_{v}$ contributes with two vertical edges of length $\left|\operatorname{ord}_{v}(f)\right|$, with inner normal $(1,0)$ and $(-1,0)$ respectively.

Next we list the edges of $R$ as its inner normal runs over $S^{1}$ : for $\theta \in S^{1}, \theta \neq$ $( \pm 1,0),(0, \pm 1)$ as we saw above, the corresponding edge has length $\sum_{v}\left\|\operatorname{ord}_{v}(\rho)\right\|$, the sum being over all $v$ such that $\operatorname{ord}_{v}(\rho) \in\left(\mathbb{R}_{>0}\right) \theta$.

For $\theta=(0,1)$, the length of the corresponding horizontal edge of $R$ equals

$$
\sum_{v: \operatorname{ord}_{v}(f)>0}-\operatorname{ord}_{v}(g)+\sum_{v: \operatorname{ord}_{v}(g)=0,-\operatorname{ord}_{v}(f)<0} \operatorname{ord}_{v}(g) .
$$

By Lemma 2.5, the corresponding edge in $-\operatorname{deg}(\rho)\left(\pi_{1}(\mathrm{~N}(C)) \times \pi_{2}(\mathrm{~N}(C))\right)$ has length $\sum_{v: \operatorname{ord}_{v}(f)>0}-\operatorname{ord}_{v}(g)$. This shows that the horizontal edge of $R$ with normal $(0,1)$ has length

$$
\sum_{v: \operatorname{ord}_{v}(g)=0,-\operatorname{ord}_{v}(f)<0} \operatorname{ord}_{v}(g)
$$

as stated. The remaining cases $(\theta=( \pm 1,0), \theta=(0,-1))$ are treated similarly.

To conclude, we use the fact that the edges of the Minkowski sum of two polygons in $\mathbb{R}^{2}$, are exactly the concatenation of the edges of the given polygons, see the discussion in [Sch93, pp. 144-145].

\section{The Generic Cases}

In this section we apply our main result to the computation of the Newton polygon in the generic cases (Corollaries 1.3, 1.4 and 1.5 in the introduction). The following result gives the degree of a generic parametrization and is an important ingredient in the proof of these corollaries.

Proposition 3.1. Let $\left(m_{1}, n_{1}\right), \ldots,\left(m_{R}, n_{R}\right) \in \mathbb{Z}^{2}$ and for $\alpha, \beta \in \mathbb{K}^{\times}$and $v_{1}, \ldots, v_{R} \in$ $\mathbb{K}$ set

$$
f(t):=\alpha \prod_{i=1}^{M}\left(t-v_{i}\right)^{m_{i}} \quad, \quad g(t):=\beta \prod_{i=1}^{M}\left(t-v_{i}\right)^{n_{i}} .
$$

If there is a pair $1 \leq i \neq j \leq R$ such that $\left(m_{i}, n_{i}\right)$ and $\left(m_{j}, n_{j}\right)$ are not linearly dependent, then the map $\mathbb{T}^{1} \rightarrow \mathbb{T}^{2}, t \mapsto(f(t), g(t))$ is birational for generic $\left(v_{i}, v_{j}\right) \in$ $\mathbb{K}^{2}$.

In particular when the vectors $\left(m_{1}, n_{1}\right), \ldots,\left(m_{R}, n_{R}\right)$ are not collinear, the parametrization defined by the functions in (3.1) is birational for generic $v_{1}, \ldots, v_{R}$. The case when all of the $\left(m_{i}, n_{i}\right)$ 's are collinear is easy to handle: we then set

$$
\left(m_{i}, n_{i}\right)=k_{i}(m, n)
$$

for some $m, n \in \mathbb{Z}$ with $\operatorname{gcd}(m, n)=1$ and $k_{i} \in \mathbb{Z} \backslash\{0\}$. Setting $h(t):=\prod_{i=1}^{R}\left(t-v_{i}\right)^{k_{i}}$ the functions in (3.1) can be written as

$$
f(t)=\alpha h(t)^{m} \quad, \quad g(t)=\beta h(t)^{n},
$$


and hence the degree of the map $\rho: t \mapsto(f(t), g(t))$ equals to the degree of $t \mapsto h(t)$ and so $\operatorname{deg}(\rho)=\eta(h)$. In particular, for generic $v_{1}, \ldots, v_{R} \in \mathbb{K}$

$$
\operatorname{deg}(\rho)=\max \left\{\sum_{i: k_{i}>0} k_{i},-\sum_{i: k_{i}<0} k_{i}\right\} .
$$

Lemma 3.2. Let $p \in \mathbb{K}(t) \backslash \mathbb{K}, q \in \mathbb{K}(t)^{\times}$and $c \in \mathbb{Z} \backslash\{0\}$, then the map

$$
\mathbb{T}^{1}-\rightarrow \mathbb{T}^{2} \quad, \quad t \mapsto\left(p(t),(t-v)^{c} q(t)\right)
$$

is birational for generic $v \in \mathbb{K}$.

Proof. Let $x_{0} \in \mathbb{T}^{1}$ generic, then the number of different solutions to $p(t)=x_{0}$ equals $\eta(p)$ and we denote these solutions $t_{1}, \ldots, t_{\eta(p)}$. As $x_{0}$ is generic, we will have $q\left(t_{i}\right), q\left(t_{j}\right) \neq 0$. Suppose that for some $i \neq j$ and almost all $v \in \mathbb{K}$ we have $\left(t_{i}-v\right)^{c} q\left(t_{i}\right)=\left(t_{j}-v\right)^{c} q\left(t_{j}\right)$ or equivalently

$$
\left(\frac{t_{j}-v}{t_{i}-v}\right)^{c}=\frac{q\left(t_{j}\right)}{q\left(t_{i}\right)}
$$

for almost all $v \in \mathbb{K}$. This implies that the rational function $v \mapsto\left(t_{j}-v\right) /\left(t_{i}-v\right)$ is constant and so $t_{i}=t_{j}$, a contradiction. We deduce then that for each $i \neq j$ the set

$$
\left\{v \in \mathbb{K}:\left(t_{i}-v\right)^{c} q\left(t_{i}\right)=\left(t_{j}-v\right)^{c} q\left(t_{j}\right)\right\}
$$

is finite and hence for $v$ outside all of these finite sets, the rational function $(t-v)^{c} q(t)$ separates the points $t_{1}, \ldots, t_{\eta(p)}$. Let $C \subset \mathbb{T}^{2}$ be the Zariski closure of the image of the map $t \mapsto\left(p(t),(t-v)^{c} q(t)\right)$ and $E_{C} \in \mathbb{K}\left[x^{ \pm 1}, y^{ \pm 1}\right]$ its defining equation, the previous considerations show that

$$
\operatorname{deg}_{y}\left(E_{C}\right)=\operatorname{Card}\left(C \cap V\left(x-x_{0}\right)\right)=\eta(p)
$$

for generic $x_{0} \in \mathbb{T}^{1}$. But on the other hand, $\eta(p) \geq 1$ because $p$ is not constant and Lemma 2.5 shows that $\operatorname{deg}_{y}\left(E_{C}\right)=\operatorname{deg}(\rho)^{-1} \eta(p)$, which concludes the proof.

Proof of proposition 3.1. Setting $\Delta:=\operatorname{det}\left(\begin{array}{cc}m_{i} & m_{j} \\ n_{i} & n_{j}\end{array}\right) \neq 0$ we have

$$
f^{n_{j}} g^{-m_{j}}=\left(t-v_{i}\right)^{\Delta} F(t) \quad, \quad f^{-n_{i}} g^{m_{i}}=\left(t-v_{j}\right)^{\Delta} G(t)
$$

for some rational functions $F, G$ not depending on the parameters $v_{i}, v_{j}$. The functions

$$
p(t):=\left(t-v_{i}\right)^{\Delta} F(t) \quad, \quad q(t):=G(t)
$$

are in the hypothesis of Lemma 3.2 , because $p \notin \mathbb{K}$ for all $v_{i} \in \mathbb{K}$ except a finite number of exceptions. For those $v_{i}$ 's, this lemma shows that the map

$$
t \mapsto\left(\left(t-v_{i}\right)^{\Delta} F(t),\left(t-v_{2}\right)^{\Delta} G(t)\right)=\left(f^{n_{j}}(t) g^{-m_{j}}(t), f^{-n_{i}}(t) g^{m_{i}}(t)\right)
$$

is birational except for a finite number of $v_{j}$ 's, and a fortiori the same is true for $t \mapsto(f(t), g(t))$.

The following result shows that for a given parametrization, the Newton polygon can be computed by using partial factorizations. This can simplify the application of Theorem 1.1 in concrete situations. 
Lemma 3.3. Let $\rho=(f, g) \in\left(\mathbb{K}(t)^{\times}\right)^{2}$ and write

$$
f(t)=\alpha \prod_{p \in \mathcal{P}} p(t)^{d_{p}} \quad, \quad g(t)=\beta \prod_{p \in \mathcal{P}} p(t)^{e_{p}}
$$

for a finite set $\mathcal{P} \subset \mathbb{K}[t]$ of pairwise coprime polynomials, $d_{p}, e_{p} \in \mathbb{N}$ and $\alpha, \beta \in \mathbb{K}^{\times}$, then

$$
\left.P\left(\left(\operatorname{ord}_{v}(\rho)\right)_{v \in \mathbb{P}^{1}}\right)=P\left(\operatorname{deg}(p)\left(d_{p}, e_{p}\right)\right)_{p \in \mathcal{P}},(\operatorname{deg}(f), \operatorname{deg}(g))\right) .
$$

Proof. Let $p \in \mathcal{P}$ and $v \in \mathbb{K}$ be a root of $p$ of multiplicity $m_{v} \geq 1$, then

$$
\operatorname{ord}_{v}(\rho)=m_{v}\left(d_{p}, e_{p}\right) \in\left(\mathbb{R}_{>0}\right)\left(d_{p}, e_{p}\right) .
$$

Hence all of the multiplicities of $\rho$ at roots of a given $p \in \mathcal{P}$ lie in the same direction and so in the construction of $P\left(\left(\operatorname{ord}_{v}(\rho)\right)_{v \in \mathbb{P}^{1}}\right)$ they concatenate together into the single vector

$$
\sum_{v \in \mathbb{K}: p(v)=0} \operatorname{ord}_{v}(\rho)=\operatorname{deg}(p)\left(d_{p}, e_{p}\right),
$$

while on the other hand $\operatorname{ord}_{\infty}(\rho)=(\operatorname{deg}(f), \operatorname{deg}(g))$. The fact that the polynomials in $\mathcal{P}$ are pairwise coprime ensures that each non-zero vector in the family $\left(\operatorname{ord}_{v}(\rho)\right)_{v \in \mathbb{P}^{1}}$ appears exactly one time as a term of $\operatorname{deg}(p)\left(d_{p}, e_{p}\right)$ for $p \in \mathcal{P}$ or as $(\operatorname{deg}(f), \operatorname{deg}(g))$, which concludes the proof.

Proof of Corollaries 1.3, 1.4 and 1.5. Corollary 1.3 is contained both in Corollary 1.4 and Corollary 1.5, so it suffices to prove these two statements.

Set $\widetilde{p}(t):=\alpha_{d}+\alpha_{d+1} t+\cdots+\alpha_{D} t^{D-d}$ and $\widetilde{q}(t):=\beta_{e}+\beta_{1} t+\cdots+\beta_{E} t^{E-e}$ so that the parametrization $\rho=(f, g)$ in Corollary 1.4 factorizes as

$$
f(t)=t^{d} \widetilde{p}(t) r(t)^{-1} \quad, \quad g(t)=t^{e} \widetilde{q}(t) r(t)^{-1} .
$$

In case $p, q, r$ are pairwise coprime, Theorem 1.1 together with Lemma 3.3 above readily imply that

$$
\begin{aligned}
\mathrm{N}\left(\rho^{*}\left(\mathbb{T}^{1}\right)\right)= & P\left(\left(\operatorname{ord}_{0}(f), \operatorname{ord}_{0}(f, g)\right),(\operatorname{deg}(\widetilde{p}), 0),(0, \operatorname{deg}(\widetilde{q})),\right. \\
& (-\operatorname{deg}(r),-\operatorname{deg}(r)),(\operatorname{deg}(f), \operatorname{deg}(g))) \\
= & P((d, e),(D-d, 0),(0, E-e),(-F,-F),(F-D, F-E)) .
\end{aligned}
$$

Reciprocally, we will show that the equality (3.5) above holds only if $p, q, r$ are pairwise coprime. To see this, note first that $\operatorname{ord}_{0}(\rho)=(d, e)$ and $\operatorname{ord}_{\infty}(\rho)=(F-D, F-E)$ because $\alpha_{d}, \alpha_{D}, \beta_{e}, \beta_{E} \neq 0$. For rational functions of the form (3.4), the only factor which might contribute to the direction $(1,0)$ is the polynomial $\widetilde{p}$ and it does contribute with the vector $(D-d, 0)$ if and only if $\widetilde{p}$ is coprime with both $r$ and $\widetilde{q}$. Similarly, the presence of the vector $(0, E-e)$ implies that $\widetilde{q}$ is coprime with both $r$ and $\widetilde{p}$ and so we conclude that $p, q, r$ are pairwise coprime.

For the second part of the statement, assume that $(D-d, 0),(0, E-e),(d, e),(F, F)$ are not collinear and for $v_{1}, v_{2}, v_{3} \in \mathbb{K}$ consider the parametrization $\rho$ associated to the specialization

$$
p(t):=t^{d}\left(t-v_{2}\right)^{D-d} \quad, \quad q(t):=t^{e}\left(t-v_{3}\right)^{E-e} \quad, \quad r(t):=\left(t-v_{4}\right)^{F} .
$$

In case $(D-d, 0),(0, E-e),(d, e)$ are not collinear, Proposition 3.1 readily implies that $\rho$ is birational for generic $v_{1}, v_{2}, v_{3}$. If this is not the case, we have that $(d, e)$ and at least one of the vectors $(D-d, 0),(0, E-e),(d, e)$ are linearly independent; 
we note $1 \leq i \leq 3$ the corresponding index. Letting $j \neq i$ another index, we apply Proposition 3.1 to the parametrization $\rho\left(t+v_{j}\right)$ and we also obtain that $\rho$ is birational for generic $v_{1}, v_{2}, v_{3}$. This readily implies that $\rho$ is birational for a generic choice of $p, q, r$ because $\operatorname{deg}(\rho)=1$ is an open condition.

The proof of Corollary 1.5 is analogous and we leave it to the interested reader.

\section{The Variety of Rational Plane Curves with Given Newton Polygon}

This section is devoted to the proof of Theorem 1.6 and more generally to the study of the variety $M_{Q}$. In particular, we show that every non-degenerate polygon is the Newton polygon of a rational plane curve. Similarly, we also determine which polygons can be the Newton polygon of a curve parameterized by polynomials or by Laurent polynomials.

Throughout this section we denote by $Q$ an arbitrary non-degenerate convex lattice polygon of $\mathbb{R}^{2}$. Setting $L:=\operatorname{Card}\left(Q \cap \mathbb{Z}^{2}\right)-1$, we identify the space of Laurent polynomials with support contained in $Q$ with $\mathbb{K}^{L+1}$ and the set of effective Weil divisors of $\mathbb{T}^{2}$ with Newton polygon contained in $Q$, with $\mathbb{P}^{L}$. As defined in the introduction, the set $M_{Q}^{\circ} \subset \mathbb{P}^{L}$ consists in the effective divisors of $\mathbb{T}^{2}$ of the form $\delta[C]$ for a rational curve $C$ and $\delta \geq 1$ such that $\delta \mathrm{N}(C)=Q$. We denote by $M_{Q} \subset \mathbb{P}^{L}$ the Zariski closure of this set.

In the sequel we construct the space of rational parametrizations corresponding to the divisors in $M_{Q}^{\circ}$. Let $\left(m_{1}, n_{1}\right), \ldots,\left(m_{r}, n_{r}\right) \in \mathbb{Z}^{2} \backslash\{(0,0)\}$ be the primitive inner normal vectors of $Q$ and $\ell_{1}, \ldots, \ell_{r} \geq 1$ the lattice length of the corresponding edge. Given a point $p_{i}=\left(p_{i, 0}: \cdots: p_{i, \ell_{i}}\right) \in \mathbb{P}^{\ell_{i}}$, we identify it with the associated monic polynomial $p_{i}(t) \in \mathbb{K}[t]$ of degree $\leq \ell_{i}$. In concrete terms,

$$
p_{i}(t):=p_{i, k}^{-1} p_{i, 0}+\cdots+p_{i, k}^{-1} p_{i, k-1} t^{k-1}+t^{k}
$$

for the maximal index $0 \leq k \leq \ell_{i}$ such that $p_{i, k} \neq 0$. We then consider the dense open subset

$$
U_{Q} \subset \mathbb{T}^{2} \times \prod_{i=1}^{r} \mathbb{P}^{\ell_{i}}
$$

of points $\left(\alpha, \beta, p_{1}, \ldots, p_{r}\right)$ such that the homogeneous associated polynomials

$$
p_{i}^{\mathrm{h}}\left(t_{0}, t_{1}\right):=t_{0}^{\ell_{i}} p_{i}\left(t_{0}^{-1} t_{1}\right) \in \mathbb{K}\left[t_{0}, t_{1}\right] \quad \text { for } 1 \leq i \leq r
$$

are pairwise coprime. To each $\boldsymbol{u}=\left(\alpha, \beta, p_{1}, \ldots, p_{r}\right) \in U_{Q}$ we associate a parametrization $\rho_{\boldsymbol{u}}=\left(f_{\boldsymbol{u}}, g_{\boldsymbol{u}}\right) \in \mathbb{K}(t)^{2}$ defined by

$$
f_{\boldsymbol{u}}(t):=\alpha \prod_{i=1}^{r} p_{i}(t)^{m_{i}} \quad, \quad g_{\boldsymbol{u}}(t):=\beta \prod_{i=1}^{r} p_{i}(t)^{n_{i}} .
$$

Lemma 4.1. The map $U_{Q} \rightarrow \mathbb{K}(t)^{2}, \boldsymbol{u} \mapsto \rho_{\boldsymbol{u}}$ is a one-to-one correspondence between $U_{Q}$ and the set of rational maps $\rho: \mathbb{T}^{1} \rightarrow \mathbb{T}^{2}$ such that $\mathrm{N}\left(\rho^{*}\left(\mathbb{T}^{1}\right)\right)=Q$.

Proof. According to Theorem 1.1, a rational map $\rho: \mathbb{T}^{1} \rightarrow \mathbb{T}^{2}$ corresponds to a member of $M_{Q}^{\circ}$ if and only if for all $v \in \mathbb{P}^{1}$ there is and index $i$ between 1 and $r$ such that $\operatorname{ord}_{v}(\rho) \in\left(\mathbb{R}_{\geq 0}\right)\left(m_{i}, n_{i}\right)$ and

$$
\sum_{v} \ell\left(\operatorname{ord}_{v}(\rho)\right)=\ell_{i} \quad \text { for } 1 \leq i \leq r,
$$


the sum being over $v \in \mathbb{P}^{1}$ such that $\operatorname{ord}_{v}(\rho) \in\left(\mathbb{R}_{>0}\right)\left(m_{i}, n_{i}\right)$. Every such map can be written as $\rho_{\boldsymbol{u}}$ for $\boldsymbol{u}=\left(\alpha, \beta, p_{1}, \ldots, p_{r}\right) \in U_{Q}$, with $(\alpha, \beta)=\operatorname{init}_{\infty}(\rho)$ and

$$
p_{i}(t)=\prod_{v \neq \infty}(t-v)^{\ell\left(\operatorname{ord}_{v}(\rho)\right)} \quad \text { for } 1 \leq i \leq r,
$$

this time the index variable runs over $v \in \mathbb{K}$ such that $\operatorname{ord}_{v}(\rho) \in\left(\mathbb{R}_{>0}\right)\left(m_{i}, n_{i}\right)$. Reciprocally, the map $\rho_{\boldsymbol{u}}$ corresponding to a point $\boldsymbol{u}=\left(\alpha, \beta, p_{1}, \ldots, p_{r}\right) \in U_{Q}$ verifies the above conditions: we have that $\operatorname{ord}_{v}\left(\rho_{\boldsymbol{u}}\right) \neq(0,0)$ if and only if $v \in V\left(p_{i}^{\mathrm{h}}\right)$ for $1 \leq i \leq r$ and in that case, $\operatorname{ord}_{v}(\rho)=\operatorname{ord}_{v}\left(p_{i}^{\mathrm{h}}\right)\left(m_{i}, n_{i}\right) \in\left(\mathbb{R}_{>0}\right)\left(m_{i}, n_{i}\right)$. Moreover

$$
\sum_{v \in V\left(p_{i}^{\mathrm{h}}\right)} \ell\left(\operatorname{ord}_{v}\left(\rho_{\boldsymbol{u}}\right)\right)=\sum_{v \in V\left(p_{i}^{\mathrm{h}}\right)} \operatorname{ord}_{v}\left(p_{i}^{\mathrm{h}}\right)=\ell_{i}
$$

and so $\mathrm{N}\left(\rho_{\boldsymbol{u}}^{*}\left(\mathbb{T}^{1}\right)\right)=Q$.

It only remains to show that the correspondence $\boldsymbol{u} \mapsto \rho_{\boldsymbol{u}}$ is injective: let $\boldsymbol{u}=$ $\left(\alpha, \beta, p_{1}, \ldots, p_{r}\right), \boldsymbol{u}^{\prime}=\left(\alpha^{\prime}, \beta^{\prime}, p_{1}^{\prime}, \ldots, p_{r}^{\prime}\right) \in U_{Q}$ such that $\rho_{\boldsymbol{u}}=\rho_{\boldsymbol{u}^{\prime}}$. For $i \neq j$ set $\Delta_{i, j}:=\operatorname{det}\left(\begin{array}{cc}m_{i} & m_{j} \\ n_{i} & n_{j}\end{array}\right) \neq 0$, so that for $1 \leq i \leq r$

$$
\alpha^{n_{i}} \beta^{-m_{i}} \prod_{j \neq i} p_{j}^{\Delta_{i, j}}=f_{\boldsymbol{u}_{i}}^{n_{i}} g_{\boldsymbol{u}}^{-m_{i}}=f_{\boldsymbol{u}^{\prime}}^{n_{i}} g_{\boldsymbol{u}^{\prime}}^{-m_{i}}=\left(\alpha^{\prime}\right)^{n_{i}}\left(\beta^{\prime}\right)^{-m_{i}} \prod_{j \neq i}\left(p_{j}^{\prime}\right)^{\Delta_{i, j}} .
$$

This shows that $p_{i}(t)$ and $p_{j}^{\prime}(t)$ are coprime for $i \neq j$ and so $p_{i}(t)=p_{i}^{\prime}(t)$ for all $i$. Moreover $(\alpha, \beta)=\operatorname{init}_{\infty}\left(\rho_{\boldsymbol{u}}\right)=\operatorname{init}_{\infty}\left(\rho_{\boldsymbol{u}^{\prime}}\right)=\left(\alpha^{\prime}, \beta^{\prime}\right)$ and we conclude that $\boldsymbol{u}=\boldsymbol{u}^{\prime}$, as desired.

Proposition 3.1 together with this Lemma readily imply that the parametrization $\rho_{\boldsymbol{u}}$ is birational for generic $\boldsymbol{u} \in U_{Q}$.

Corollary 4.2. Let $Q$ be a non-degenerate convex lattice polygon, then $\operatorname{deg}\left(\rho_{\boldsymbol{u}}\right)=1$ for $\boldsymbol{u}$ in a dense open subset of $U_{Q}$.

Different parametrizations might define the same push-forward cycle. To avoid most of this redundancy, we introduce an equivalence relation by agreeing that two parametrizations $\rho, \rho^{\prime}: \mathbb{T}^{1} \rightarrow \mathbb{T}^{2}$ are equivalent if there exists a birational automorphism $\mu$ of $\mathbb{T}^{1}$ such that $\rho^{\prime}=\rho \circ \mu$. The birational automorphisms of $\mathbb{T}^{1}$ are the Möbius transformations, that is the maps of the form $\mathbb{T}^{1} \rightarrow \mathbb{T}^{1}, t \mapsto \frac{\alpha t+\beta}{\gamma t+\delta}$ for some $\alpha, \beta, \gamma, \delta \in$ $\mathbb{K}$ such that $\alpha \delta-\beta \gamma \neq 0$. We then set

$$
P_{Q}:=U_{Q} / \sim
$$

for the space of parametrizations of effective divisors in $M_{Q}^{\circ}$ modulo equivalence. The assumption that $Q$ is non-degenerate implies that it has at least three different edges, that is $r \geq 3$. Given any three different points of $\mathbb{P}^{1}$, there is a unique Möbius transformation which brings them to $0,1, \infty$ respectively. We can thus fix a system of representatives of $P_{Q}$ as the set of points $\boldsymbol{u}=\left(\alpha, \beta, p_{1}, \ldots, p_{r}\right) \in U_{Q}$ such that the polynomials $p_{1}(t), p_{2}(t)$ and $p_{3}(t)$ have respectively 0,1 and $\infty$ as a root. This identifies $P_{Q}$ with a subset of $U_{Q}$. We can also verify that $P_{Q}$ is linearly isomorphic to a dense open subset of

$$
\mathbb{T}^{2} \times \prod_{i=1}^{3} \mathbb{P}^{\ell_{i}-1} \times \prod_{i=4}^{r} \mathbb{P}^{\ell_{i}}
$$


It is well-known that the equation of the cycle $\rho^{*}\left(\mathbb{T}^{1}\right)$ can be written in terms of the Sylvester resultant. Set

$$
D:=\sum_{i: m_{i}>0} \ell_{i} m_{i}=-\sum_{i: m_{i}<0} \ell_{i} m_{i} \quad, \quad E:=\sum_{i: n_{i}>0} \ell_{i} n_{i}=-\sum_{i: n_{i}<0} \ell_{i} n_{i},
$$

these equalities being a consequence of the balancing condition $\sum_{i=1}^{r} \ell_{i}\left(m_{i}, n_{i}\right)=$ $(0,0)$. We then consider the map $U_{Q} \rightarrow \mathbb{K}\left[x^{ \pm 1}, y^{ \pm 1}\right]$ defined by

$$
\rho_{\boldsymbol{u}}=(f, g) \mapsto x^{a} y^{b} \operatorname{Res}_{D, E}\left(f_{\mathrm{den}}(t) x-f_{\text {num }}(t), g_{\text {den }}(t) y-g_{\text {num }}(t) ; t\right)
$$

where $\operatorname{Res}_{D, E}$ stands for the Sylvester resultant two univariate polynomials of degree $D$ and $E$ respectively and $a, b \in \mathbb{Z}$ are maximal so that $Q-(a, b) \in\left(\mathbb{R}_{>0}\right)^{2}$. Note that for $\boldsymbol{u} \in U_{Q}$ we have $\eta\left(f_{\boldsymbol{u}}\right)=D$ and $\eta\left(g_{\boldsymbol{u}}\right)=E$ and so

$$
\operatorname{deg}_{t}\left(f_{\text {den }}(t) x-f_{\text {num }}(t)\right)=D \quad, \quad \operatorname{deg}_{t}\left(g_{\text {den }}(t) y-g_{\text {num }}(t)\right)=E,
$$

hence the map (4.2) is well-defined on the whole of $U_{Q}$.

Proposition 4.3. With the above notation, Formula (4.2) induces a surjective regular map $\Xi: P_{Q} \rightarrow M_{Q}^{\circ}$. The fiber of $\Xi$ at $\delta[C] \in M_{Q}^{\circ}$ is an irreducible variety of dimension $2 \delta-2$.

Proof. Recall that for univariate polynomials $p, q$ of degree bounded by $D$ and $E$ respectively, the Sylvester resultant $\operatorname{Res}_{D, E}(p, q)$ vanishes if and only if $\operatorname{gcd}(p, q) \neq 1$, or $\operatorname{deg}(p)<D$ and $\operatorname{deg}(q)<E$. Put

$$
R_{\boldsymbol{u}}(x, y):=\operatorname{Res}_{D, E}\left(f_{\mathrm{den}}(t) x-f_{\text {num }}(t), g_{\mathrm{den}}(t) y-g_{\mathrm{num}}(t) ; t\right) \in \mathbb{K}[x, y] .
$$

In our setting we have that $\operatorname{deg}_{t}\left(f_{\operatorname{den}}(t) x-f_{\text {num }}(t)\right)=D$ and $\operatorname{deg}_{t}\left(g_{\text {den }}(t) y-g_{\text {num }}(t)\right)=$ $E$ and they are coprime as polynomials in $\mathbb{K}(x, y)[t]$. This implies that $R_{\boldsymbol{u}} \neq 0$ and moreover $R_{\boldsymbol{u}}(0, y), R_{\boldsymbol{u}}(x, 0) \neq 0$. We can conclude then that $R_{\boldsymbol{u}}$ is is a defining equation for the divisor $\delta[C]$, which is a Taylor polynomial neither divisible by $x$ nor by $y$. Hence $\mathrm{N}\left(R_{\boldsymbol{u}}\right)=Q-(a, b)$ and this is equivalent to $x^{a} y^{b} R_{\boldsymbol{u}} \in M_{Q}^{\circ}$.

This shows that Formula (4.2) defines a regular surjective map from $U_{Q}$ onto $M_{Q}^{\circ}$. This map is well-defined in the quotient space $P_{Q}$ because it only depends on the push-forward cycle $\rho^{*}\left(\mathbb{T}^{1}\right)$ and moreover, it is regular on this quotient space because $P_{Q}$ can be regarded as a subset of $U_{Q}$.

For a given divisor $\delta[C] \in M_{Q}^{\circ}$ set

$$
\Omega_{\boldsymbol{u}}:=\left\{\boldsymbol{u}: \mathrm{N}\left(\rho_{\boldsymbol{u}}^{*}\left(\mathbb{T}^{1}\right)\right)=\delta[C]\right\} \subset U_{Q} .
$$

By Lüroth theorem Wal50, $\S$ V.7, pp. 149-151 there exists a birational map $\psi$ : $\mathbb{T}^{1} \rightarrow C$, and so $\Omega_{\boldsymbol{u}}$ is isomorphic to the space of birational endomorphism of $\mathbb{T}^{1}$ of degree $\delta$ through the map

$$
\operatorname{End}\left(\mathbb{T}^{1} ; \delta\right) \rightarrow \Omega_{\boldsymbol{u}} \quad, \quad h \mapsto \psi \circ h,
$$

with inverse $\rho \mapsto \psi^{-1} \circ \rho$. The group $\operatorname{Aut}\left(\mathbb{T}^{1}\right)$ of Möbius transformations acts on $\operatorname{End}\left(\mathbb{T}^{1} ; \delta\right)$ by right composition and the fiber $\Xi^{-1}(\delta[C]) \subset P_{Q}$ identifies with the set-theoretical quotient $\operatorname{End}\left(\mathbb{T}^{1} ; \delta\right) / \operatorname{Aut}\left(\mathbb{T}^{1}\right)$.

In case $\delta=1$ we have that $\operatorname{End}\left(\mathbb{T}^{1} ; 1\right)=\operatorname{Aut}\left(\mathbb{T}^{1}\right)$ so in this case the quotient is a point. For $\delta \geq 2$, we affirm that the orbit of a generic element $h \in \operatorname{End}\left(\mathbb{T}^{1} ; \delta\right)$ is isomorphic to $\operatorname{Aut}\left(\mathbb{T}^{1}\right)$ : if we have $h=h \circ \mu$ for $\mu \in \operatorname{Aut}\left(\mathbb{T}^{1}\right)$, the Möbius transformation $\mu$ must 
fix the set of zeros and poles of $h$. This set has at least four generic points of $\mathbb{T}^{1}$ because $h$ is generic and $\delta \geq 2$, and so $\mu=\operatorname{Id}_{\mathbb{T}}^{1}$. Hence the fiber of

$$
\pi: \operatorname{End}\left(\mathbb{T}^{1} ; \delta\right) \rightarrow \operatorname{End}\left(\mathbb{T}^{1} ; \delta\right) / \operatorname{Aut}\left(\mathbb{T}^{1}\right)
$$

over a generic point $w$ has dimension 3, and so by the theorem of dimension of fibers

$$
\operatorname{dim}\left(\operatorname{End}\left(\mathbb{T}^{1} ; \delta\right) / \operatorname{Aut}\left(\mathbb{T}^{1}\right)\right)=\operatorname{dim}\left(\operatorname{End}\left(\mathbb{T}^{1} ; \delta\right)\right)-\operatorname{dim} \pi^{-1}(w)=(2 \delta+1)-3,
$$

which concludes the proof.

From the proof above, we see that the fiber $\Xi^{-1}(\delta[C])$ is in fact isomorphic to the quotient space $\operatorname{End}\left(\mathbb{T}^{1} ; \delta\right) / \operatorname{Aut}\left(\mathbb{T}^{1}\right)$.

Corollary 4.4. The set of reduced divisors in $M_{Q}^{\circ}$ contains a dense open subset. In particular, $\Xi$ is a bijection between some dense open subsets of $P_{Q}$ and $M_{Q}^{\circ}$.

This is a direct consequence of Corollary 4.2 and the fact that the map $\Xi: P_{Q} \rightarrow M_{Q}^{\circ}$ is dominant. We are now in position to prove Theorem 1.6.

Proof of Theorem 1.6. Set $\ell:=\sum_{i=1}^{r} \ell_{i}=\operatorname{Card}\left(\partial Q \cap \mathbb{Z}^{2}\right)$. By Corollary 4.4, we have that $\Xi$ is a bijection between some dense open subsets of $P_{Q}$ and $M_{Q}^{\circ}$ and so the theorem of dimension of fibers implies that

$$
\operatorname{dim}\left(M_{Q}\right)=\operatorname{dim}\left(P_{Q}\right)=2+\sum_{i=1}^{3}\left(\ell_{i}-1\right)+\sum_{i=4}^{r} \ell_{i}=\ell-1 .
$$

In particular $M_{Q}$ is unirational, because $P_{Q}$ is birational to $\mathbb{T}^{2} \times \prod_{i=1}^{3} \mathbb{P}^{\ell_{i}-1} \times \prod_{i=4}^{r} \mathbb{P}^{\ell_{i}}$ and a fortiori to $\mathbb{A}^{\ell-1}$.

In case $\operatorname{char}(\mathbb{K})=0$, the field extension $\Xi^{*}\left(\mathbb{K}\left(M_{Q}\right)\right) \subset \mathbb{K}\left(\mathbb{A}^{\ell-1}\right)$ is separable and so its degree equals the cardinality of a generic fiber [Sha94, § II.6.3, pp. 142-145]. Hence $\Xi^{*}\left(\mathbb{K}\left(M_{Q}\right)\right)=\mathbb{K}\left(\mathbb{A}^{\ell-1}\right)$ and in particular $M_{Q}$ is rational.

Set $c(Q)$ for the content of $Q$, defined as the maximal integer $c \geq 1$ such that $c^{-1} Q$ is a lattice polygon modulo a translation. The polygon $Q$ is non-contractible whenever $c(Q)=1$.

For $\delta[C] \in M_{Q}^{\circ}$ we have

$$
Q=\mathrm{N}(\delta[C])=\delta \mathrm{N}(C)
$$

and so $\delta \mid c(Q)$ because $\mathrm{N}(C)$ is a lattice polygon. Hence the possible multiplicities of a divisor in $M_{Q}^{\circ}$ are exactly the divisors of $c(Q)$. In particular, $\Xi: P_{Q} \rightarrow M_{Q}^{\circ}$ is a bijection if and only if $Q$ is non-contractible.

Alternatively, the content of $Q$ coincides with the greatest common divisor of the lattice length of its edges. Hence in the setting of Corollary 4.2, for $\boldsymbol{u} \in U_{Q}$ we have $\operatorname{deg}\left(\rho_{\boldsymbol{u}}\right) \mid \operatorname{gcd}\left(\ell_{1}, \ldots, \ell_{r}\right)$. In particular, $\rho_{\boldsymbol{u}}$ is birational for all $\boldsymbol{u} \in U_{Q}$ if and only if $\operatorname{gcd}\left(\ell_{1}, \ldots, \ell_{r}\right)=1$.

The following example shows that in positive characteristic, the map $\Xi$ can be generically ramified.

Example 4.5. Consider the polygon $Q$ in Figure 4 below. Its primitive inner normals are the vectors $(1,2),(-2,-1),(1,-1)$. This is a non-contractible polygon and so all of the divisors in $M_{Q}^{\circ}$ are reduced. A parametrization of a rational plane curve with 

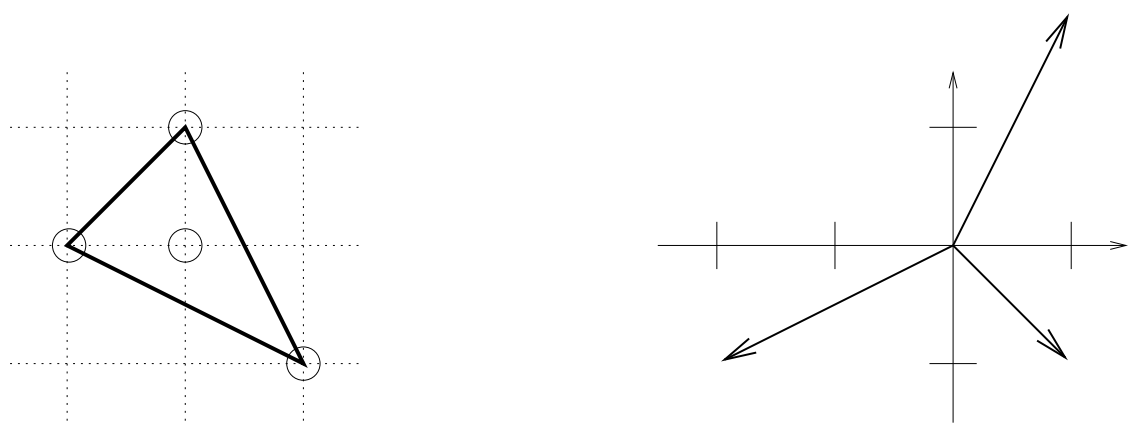

FIgURE 4. A reflexive polygon and its primitive inner normals

Newton polygon $Q$ is equivalent modulo a Möbius transformation to one of the form $\rho=(f, g)$ with

$$
f=\alpha t(t-1) \quad, \quad g=\beta \frac{t^{2}}{t-1}
$$

for some $\alpha, \beta \in \mathbb{K}^{\times}$. Hence $P_{Q}=\mathbb{T}^{2}$ and we have that

$$
\operatorname{Res}_{2,2}\left(x-\alpha t(t-1),(t-1) y-\beta t^{2} ; t\right)=\beta^{2} x^{2}+3 \alpha \beta x y-\alpha x y^{2}+\alpha^{2} \beta y .
$$

The map $\Xi$ then explicites as

$$
\Xi: \mathbb{T}^{2} \rightarrow \mathbb{P}^{3} \quad, \quad(\alpha, \beta) \mapsto\left(\beta^{2}: 3 \alpha \beta:-\alpha: \alpha^{2} \beta\right) .
$$

Let $z_{x^{2}}, z_{x y}, z_{x y^{2}}, z_{y}$ denote the homogeneous coordinates of $\mathbb{P}^{3}$, corresponding to the monomials whose exponents are the lattice points of $Q$. Then we have that $M_{Q}$ is a surface in $\mathbb{P}^{3}$ with homogeneous defining equation

$$
z_{x y}^{3}-27 z_{x^{2}} z_{x y^{2}} z_{y}=0 .
$$

If $\operatorname{char}(\mathbb{K}) \neq 3$, this is a smooth toric surface of degree 3 . On the other hand, for $\operatorname{char}(\mathbb{K})=3$ the map $\Xi$ is generically ramified of degree 3 . The defining equation of $M_{Q}$ is $z_{x y}=0$, hence it is a rational surface, but of degree 1 .

In all the examples computed so far, the variety $M_{Q}$ is always rational independently of the characteristic of the base field $\mathbb{K}$. It would be interesting to determine if this holds in general. This does not follow immediately from Lemma 4.3, as in positive characteristic there are examples of non-rational surfaces whose field of fractions admits a purely inseparable extension Shi74.

Example 4.6. Consider the polygon $\widetilde{Q}$ in the figure below:
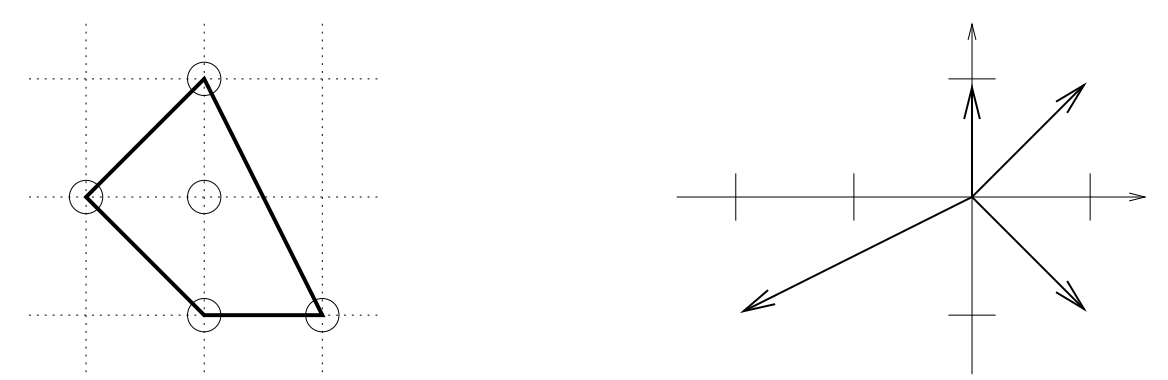

FIGURE 5. A second reflexive polygon and its primitive inner normals 
Its inner normals are the vectors $(1,1),(0,1),(-2,-1),(1,-1)$. Hence a parametrization of a rational plane curve with this Newton polygon is equivalent modulo a Möbius transformation to one of the form $\rho=(f, g)$ with

$$
f=\alpha t(t-1) \quad, \quad g=\beta \frac{t(t-v)}{t-1}
$$

for some $\alpha, \beta \in \mathbb{K}^{\times}$and $v \in \mathbb{K} \backslash\{0,1\}$. Let $z_{x^{2}}, z_{x y}, z_{x y^{2}}, z_{x}, z_{y}$ denote the homogeneous coordinates of $\mathbb{P}^{3}$, corresponding to the monomials whose exponents are the lattice points in $\widetilde{Q}$. We have that $P_{\widetilde{Q}}=\mathbb{T}^{2} \times(\mathbb{K} \backslash\{0,1\})$. By computing the corresponding resultant, the map $\Xi$ expresses as

$$
\begin{aligned}
\Xi & : \mathbb{T}^{2} \times(\mathbb{K} \backslash\{0,1\}) \rightarrow \mathbb{P}^{4} \\
\quad(\alpha, \beta, v) & \mapsto\left(\beta^{2}: \alpha \beta(3-2 v):-\alpha: \alpha \beta^{2} v(v-1): \alpha^{2} \beta(1-v)\right) .
\end{aligned}
$$

In characteristic 0 , the variety $M_{\widetilde{Q}}$ is a hypersurface of $\mathbb{P}^{4}$ of degree 5 with homogeneous defining equation

$-z_{y} z_{x^{2}} z_{x y}^{3}+z_{x} z_{x y}^{4}-8 z_{x}^{2} z_{x y^{2}} z_{x y}^{2}+16 z_{x}^{3} z_{x y^{2}}^{2}-27 z_{y}^{2} z_{x y^{2}} z_{x^{2}}^{2}+36 z_{x} z_{x y^{2}} z_{y} z_{x^{2}} z_{x y}=0$.

Its singular locus contains the surface defined by the equations

$$
27 z_{y} z_{x^{2}} z_{x y^{2}}+2 z_{x, y}^{3}=0 \quad, \quad 12 z_{x} z_{x y^{2}}+z_{x y}^{2}
$$

together with two lines and a conic.

For a given polygon $Q$, the map $\Xi$ is regular in principle only on $U_{Q}$. It might be interesting to study what happens when we get to the border of $U_{Q}$ and more generally, to make explicit the Zariski closure of $M_{Q}$ in geometric or combinatorial terms.

In this direction, the behavior of $\Xi$ when a root of $p_{i}$ concides with one of $p_{j}$ in (4.1) for some $i \neq j$ has a nice combinatorial description. For instance, if we set $v=0$ in (4.3), we obtain the parametrization in Example 4.5. This gives a natural inclusion

$$
M_{Q} \hookrightarrow M_{\widetilde{Q}}
$$

which corresponds to a shrinking of the polygon $\widetilde{Q}$ into the polygon $Q$ from Example 4.5, as shown in Figure 6.

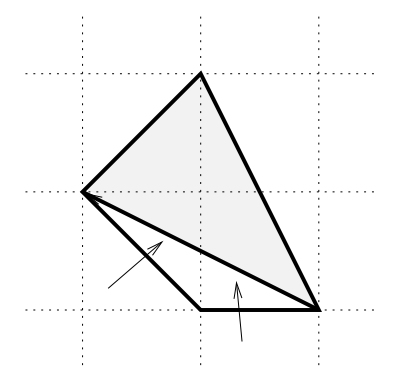

Figure 6. Polygon $\widetilde{Q}$ shrinks into polygon $Q$

This can be explained by the merging of the primitive inner normals $(1,1)$ and $(0,1)$ into its sum $(1,2)$. These observations can be extended to other cases when a root of $p_{i}$ coincides with one of $p_{j}$ for $i \neq j$, but does not seem to suffice to explain all of the points in $M_{Q} \backslash M_{Q}^{\circ}$.

The following result follows immediately from Theorem 1.6. 
Corollary 4.7. Let $Q \subset \mathbb{R}^{2}$ be a non degenerate convex lattice polygon, then $M_{Q}=\mathbb{P}^{L}$ if and only if $Q$ has no interior points, and $M_{Q}$ is a hypersurface if and only if $Q$ has exactly one interior point.

The first part of this corollary is also a consequence of Baker's formula for the geometric genus of a curve in a toric surface [Bak1893, Kho78, BS07, as this formula implies that the geometric genus of a generic plane curve with Newton polygon $Q$ equals $\operatorname{Card}\left(Q^{\circ} \cap \mathbb{Z}^{2}\right)$.

Convex lattice polygons without interior points can be easily classified. There is an infinite number of them, and modulo lattice equivalence they are either of the form Conv $((0,0),(0,2),(2,0))$ or

$$
\operatorname{Conv}((0,0),(0,1),(a, 1),(b, 0))
$$

with $a \geq 0$ and $b \geq 1$ such that $a \leq b$.

Polygons with exactly one interior point have nice properties and have been extensively studied. A polygon has exactly one interior point if and only if it is reflexive in the sense that its polar polygon is again a lattice polygon. Reflexive polygons are important because they exactly correspond to (non necessarily smooth) Fano toric surfaces. There are 16 reflexive polygons modulo lattice equivalence and pictures of them can be found for instance in [PV00]. We used three of these polygons in our examples 1.2 , 4.5 and 4.6 .

A further natural question in this context is whether for a rational plane curve with given polygon $Q$, all of the monomials associated to the lattice points in $Q$ appear. In general, the answer is "no" even for the generic member of $M_{Q}^{\circ}$. In characteristic 3, Example 4.5 actually shows that an equation with that Newton polygon defines a rational curve if and only if the coefficient of the monomial $x y$ is zero. It would be interesting to determine whether one can produce a similar example in characteristic 0 . Note that the fact that the equation of the generic member of $M_{Q}$ does not depend on the monomial $x^{a} y^{b}$ for a given $(a, b) \in Q$ is equivalent to say that $M_{Q}$ is contained in the standard hyperplane $V\left(z_{x^{a} y^{b}}\right)$ of $\mathbb{P}^{L}$.

It would also be interesting to determine the degree of $M_{Q}$ as a projective variety and its singular locus.

Finally we prove Theorem 1.7 on the shape of the Newton polygon of plane curves parameterized by polynomials or by Laurent polynomials.

Proof of Theorem 1.7. Let $Q$ be a non-degenerate convex lattice polygon such that $Q=\mathrm{N}\left(\overline{\rho\left(\mathbb{T}^{1}\right)}\right)$ for $\rho \in \mathbb{K}[t]^{2}$. Setting $\delta:=\operatorname{deg}(\rho)$ we have $\delta Q=\mathrm{N}\left(\rho^{*}\left(\mathbb{T}^{1}\right)\right)$ and this holds if and only if $\rho$ corresponds to a point $\boldsymbol{u}=\left(\alpha, \beta, p_{1}, \ldots, p_{r}\right) \in U_{\delta Q}$ with $p_{i}(t)=1$ whenever $\left(m_{i}, n_{i}\right) \notin \mathbb{N}^{2}$. Let $i_{0}$ be any of such indexes. We have then $V\left(p_{i_{0}}^{\mathrm{h}}\right)=\{\infty\}$, and there is at most one of these indexes with this property, because the polynomials $p_{1}^{\mathrm{h}}, \ldots, p_{r}^{\mathrm{h}}$ are pairwise coprime.

On the other hand, not all of the $\left(m_{i}, n_{i}\right)$ 's can lie in $\mathbb{N}^{2}$ because of the balancing condition and so there is exactly one such index. We conclude that $\left(\mathbb{R}_{>0}\right)\left(m_{i_{0}}, n_{i_{0}}\right)$ is the only inner normal direction of $Q$ which does not lie in $\left(\mathbb{R}_{\geq 0}\right)^{2}$.

Conversely, assume that $Q$ has exactly one inner normal direction not lying in $\left(\mathbb{R}_{\geq 0}\right)^{2}$. Then all of the points $\boldsymbol{u}=\left(\alpha, \beta, p_{1}, \ldots, p_{r}\right) \in U_{Q}$ such that $p_{i_{0}}(t)=1$ for the index $i_{0}$ corresponding to that inner direction, give parametrizations $\rho_{\boldsymbol{u}}$ such that $\mathrm{N}\left(\rho_{\boldsymbol{u}}^{*}\left(\mathbb{T}^{1}\right)\right)=$ 
$Q$, By Proposition 3.1 the map $\rho_{\boldsymbol{u}}$ is birational for $\boldsymbol{u}$ generic within the considered points and hence $Q=\mathrm{N}\left(\overline{\rho_{\boldsymbol{u}}\left(\mathbb{T}^{1}\right)}\right)$ for such $\boldsymbol{u}$ 's.

Similarly, $Q=\mathrm{N}\left(\overline{\rho\left(\mathbb{T}^{1}\right)}\right)$ for $\rho \in \mathbb{K}\left[t^{ \pm 1}\right]^{2}$ if and only if $\rho$ corresponds to a point $\boldsymbol{u}=\left(\alpha, \beta, p_{1}, \ldots, p_{r}\right) \in U_{\delta Q}$ such that $p_{i}(t)=t^{a(i)}$ for $a(i) \in \mathbb{N}$ whenever $\left(m_{i}, n_{i}\right) \notin \mathbb{N}^{2}$. For such an index $i_{0}$ we have that $V\left(p_{i_{0}}^{\mathrm{h}}\right) \subset\{0, \infty\}$ and so there are at most two such indexes. As before, the balancing condition shows that there must be at least one such index. We conclude that in this case there are one or two inner normal directions of $Q$ which do not lie in $\left(\mathbb{R}_{\geq 0}\right)^{2}$. The converse is proved with the same strategy of the previous case.

4.1. The degenerate case. We consider separately the case when $Q$ is a lattice segment, because the corresponding results are slightly different. If this is the case, modulo a translation we have

$$
Q=\operatorname{Conv}((0,0),(k a, k b)) \subset \mathbb{R}^{2}
$$

for a primitive vector $(a, b) \in \mathbb{Z}^{2}$ and $k:=\ell(Q) \geq 1$. The general Laurent polynomial with Newton polygon $Q$ is of the form $F(x, y)=p\left(x^{a} y^{b}\right)$ for a polynomial $p(s)=$ $p_{0}+p_{1} s+\cdots+p_{k} s^{k}$ such that $p_{0}, p_{k} \neq 0$. Hence

$$
V(F)=\bigcup_{\xi \in V(p)} V\left(x^{a} y^{b}-\xi\right)
$$

Each component curve $V\left(x^{a} y^{b}-\xi\right)$ is rational, as it can be parameterized by a map $\mathbb{T}^{1} \rightarrow \mathbb{T}^{2}, t \mapsto\left(\xi^{c} t^{b}, \xi^{d} t^{-b}\right)$ for integers $c, d$ such that $c a+d b=1$. Hence $F \in M_{Q}^{\circ}$ if and only if $p$ has only one root in $\mathbb{T}^{1}$, or equivalently if and only if $F(x, y)=\nu\left(x^{a} y^{b}-\xi\right)^{k}$ for some $\nu, \xi \in \mathbb{K}^{\times}$. We deduce that

$$
M_{Q}^{\circ}=\left\{\left(\left(\begin{array}{c}
k \\
j
\end{array}\right) t^{j}: 0 \leq j \leq k\right): t \in \mathbb{T}^{1}\right\} \subset \mathbb{P}^{k},
$$

which shows that $M_{Q}$ is linearly isomorphic to the Veronese curve in case char $(\mathbb{K})=$ 0 or $>k$, and linearly isomorphic to a projection of this Veronese curve, in any characteristic. Thus the following is the analog of Theorem 1.6 in our setting.

Proposition 4.8. Let $Q \subset \mathbb{R}^{2}$ be a lattice segment, then $M_{Q}$ is a rational curve.

Note that this dimension 1 of $M_{Q}$ can be interpreted as the number of points in the relative boundary of $Q$ minus 1 .

On the other hand, the multiplicity of all of the divisors in $M_{Q}^{\circ}$ equals $\ell(Q)$, as they are of the form $F(x, y)=\nu\left(x^{a} y^{b}-\xi\right)^{k}$ for some $\nu, \xi \in \mathbb{K}^{\times}$. Hence the lattice segment $Q$ realizes as the Newton polygon of a rational plane curve if and only if $\ell(Q)=1$.

\section{REFERENCES}

[Bak1893] H.F. Baker, Examples of applications of Newton's polygon to the theory of singular points of algebraic functions. Trans. Cambridge Phil. Soc. 15 (1893) 403-450.

[BS07] T. Beck, J. Schicho, Parametrization of algebraic curves defined by sparse equations. Appl. Algebra Engrg. Comm. Comput. 18 (2007) 127-150.

[Ber75] D.N. Bernštein, The number of roots of a system of equations (in Russian). Funk. Anal. Priloz. 9 (1975) 1-4; English translation in Functional Anal. Appl. 9 (1975) 183-185.

[EKP07] I. Emiris, C. Konaxis, L. Palios, Computing the Newton polytope of specialized resultants. Proceeding of the MEGA 2007 conference, 27 pp.. 
[EK03] I. Emiris, I. Kotsireas, Implicitization with polynomial support optimized for sparseness. Lecture Notes in Comput. Sci. 2669 (2003) 397-406, Springer.

[EK05] I. Emiris, I. Kotsireas, Implicitization exploiting sparseness. DIMACS Ser. Discrete Math. Theoret. Comput. Sci. 67 (2005) 281-298, Amer. Math. Soc..

[EKho07] A. Esterov, A. Khovanskiŭ, Elimination theory and Newton polytopes. To appear in Functional Analysis and Other Mathematics. E-print arxiv:math/0611107, 34 pp..

[Fra06] M. Franz, Convex: a Maple package for convex geometry, version 1.1. Available at http://www-fourier.ujf-grenoble.fr/ franz/convex/

[Jel05] Z. Jelonek, On the effective Nullstellensatz. Invent. Math. 162 (2005) 1-17.

[Kho78] A. Khovanskiǔ, Newton polyhedra, and the genus of complete intersections. Funk. Anal. i Priloz. 12 (1978) 51-61; English translation in Functional Anal. Appl. 12 (1978) 38-46.

[McM04] P. McMullen, Mixed fibre polytopes. Discrete Comput. Geom. 32 (2004) 521-532.

[PS04] P. Philippon, M. Sombra, Hauteur normalisée des variétés toriques projectives. To appear in J. Inst. Math. Jussieu. E-print arxiv:math/0406476, 38pp..

[PS07a] P. Philippon, M. Sombra, Une nouvelle majoration pour le nombre de solutions d'un système d'équations polynomiales. C. R. Acad. Sci. Paris 345 (2007) 335-340.

[PS07b] P. Philippon, M. Sombra, A refinement of the Kušnirenko-Bernštein estimate. E-print arXiv:0709.3306, 45 pp..

[PV00] B. Poonen, F. Rodríguez-Villegas, Lattice polygons and the number 12. Amer. Math. Monthly 107 (2000) 238-250.

[Sch93] R. Schneider, Convex bodies: the Brunn-Minkowski theory. Cambridge Univ. Press, 1993.

[Sha94] I.R. Shafarevich, Basic algebraic geometry. 1. Varieties in projective space. Second edition. Springer, 1994.

[Shi74] T. Shioda, An example of unirational surfaces in characteristic p. Math. Annalen 211 (1974) 233-236.

[ST07] B. Sturmfels, J. Tevelev, Elimination theory for tropical varieties. E-print arXiv:0704.3471, 19 pp.

[STY07] B. Sturmfels, J. Tevelev, J. Yu, The Newton polytope of the implicit equation. Moscow Math. J. 7 (2007) 327-346.

[SY07] B. Sturmfels, J. Yu, Tropical implicitization and mixed fiber polytopes. To appear in Software for Algebraic Geometry, IMA Volumes in Mathematics and its Applications, Springer. E-print arXiv:0706.0564, $20 \mathrm{pp.}$

[SY94] B. Sturmfels, J.-T. Yu, Minimal polynomials and sparse resultants. In Zero-dimensional schemes (Ravello, 1992) 317-324, De Gruyter, 1994.

[Wal50] R.J. Walker, Algebraic Curves. Princeton Univ. Press, 1950.

Universitat de Barcelona, Departament d’Àlgebra i Geometria. Gran Via 585, 08007 BARCElona, Spain.

E-mail address: cdandrea@ub.edu

$U R L:$ http://carlos.dandrea.name/

Universitat de Barcelona, Departament d’Àlgebra i Geometria. Gran Via 585, 08007 Barcelona, Spain.

E-mail address: sombra@ub.edu

$U R L$ : http://atlas.mat.ub.es/personals/sombra/ 